
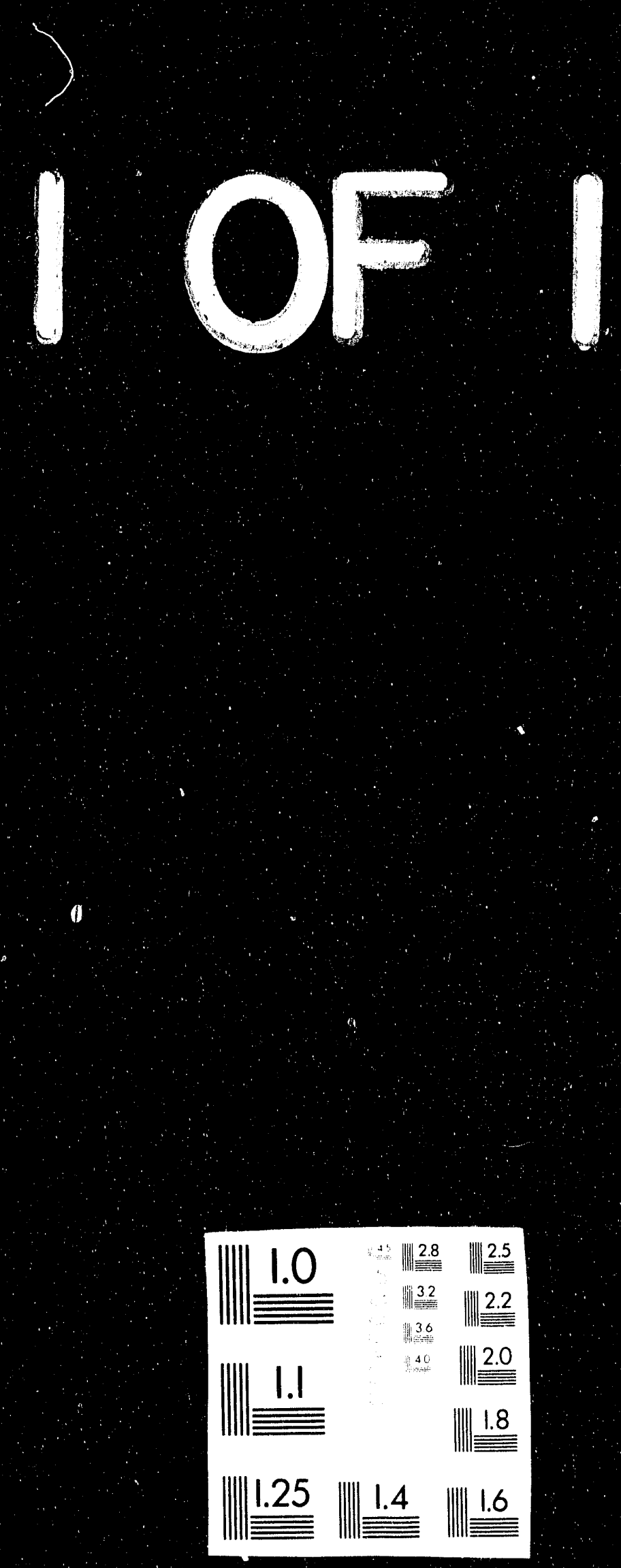


\title{
TRITIUM PERMEATION MODEL FOR PLASMA FACING COMPONENTS
}

\author{
G. R. Longhurst \\ Published December 1992 \\ Idaho National Engineering Laboratory \\ EG\&G Idaho, Inc. \\ Idaho Falls, Idaho 83415
}

Prepared for the U.S. Department of Energy Office of Fusion Enorgy Under DOE Flold Office, Idaho Contract DE-AC07-76ID0150 


\begin{abstract}
This report documents the development of a simplified one-dimensional tritium permeation and retention model. The model makes use of the same physical mechanisms as more sophisticated, time-transient codes such as implantation, recombination, diffusion, trapping and thermal gradient effects. It takes advantage of a number of simplifications and approximations to solve the steady-state problem and then provides interpolating functions to make estimates of intermediate states based on the steady-state solution. The model is developed for solution using commercial spread-sheet software such as Lotus $123 \mathrm{Tm}$. Comparison calculations are provided with the verified and validated TMAP4 transient code with good agreement. Results of calculations for the ITER CDA divertor are also included.
\end{abstract}




\section{CONTENTS}

ABSTRACT $\ldots \ldots \ldots \ldots \ldots \ldots \ldots \ldots \ldots \ldots \ldots \ldots \ldots \ldots \ldots \ldots \ldots \ldots$

CONTENTS $\ldots \ldots \ldots \ldots \ldots \ldots \ldots \ldots \ldots \ldots \ldots \ldots \ldots \ldots \ldots \ldots$ iii

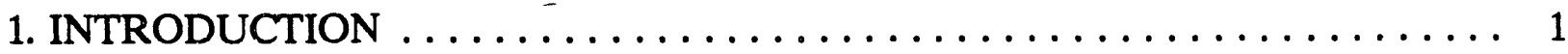

2. MODEL DESCRIPTION $\ldots \ldots \ldots \ldots \ldots \ldots \ldots \ldots \ldots \ldots \ldots \ldots \ldots \ldots$

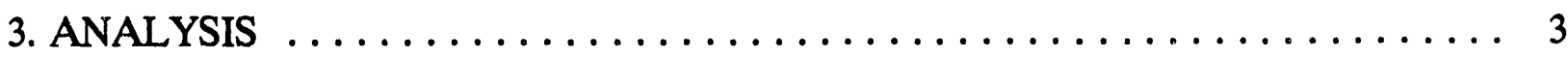

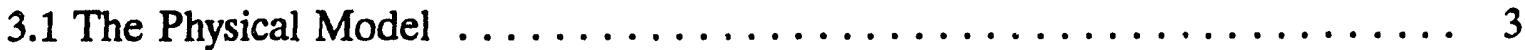

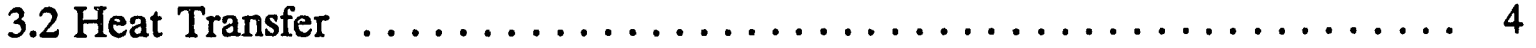

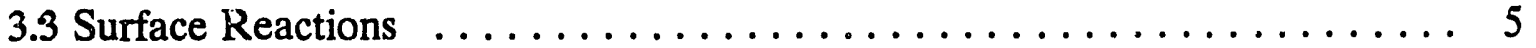

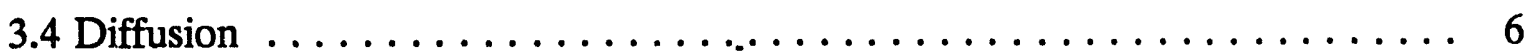

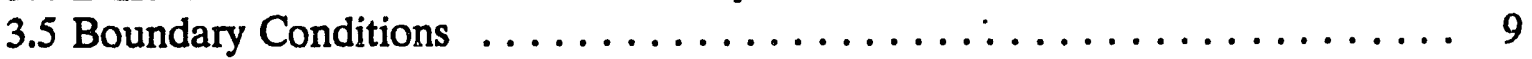

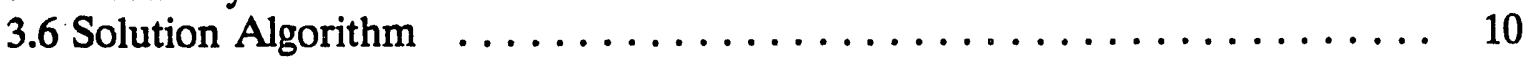

4. INVENTORY AND PERMEATION $\ldots \ldots \ldots \ldots \ldots \ldots \ldots \ldots \ldots \ldots \ldots \ldots$

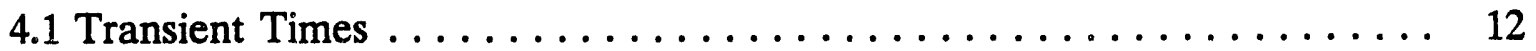

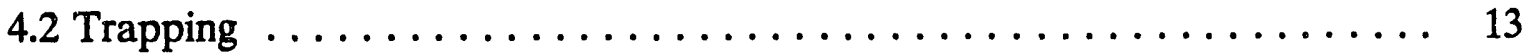

4.3 Surface Effects . . . . . . . . . . . . . . . . . . 15

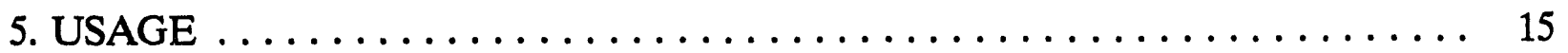

6. COMPARISON CALCULATIONS $\ldots \ldots \ldots \ldots \ldots \ldots \ldots \ldots$

7. ITER DIVERTOR CALCULATIONS $\ldots \ldots \ldots \ldots \ldots \ldots \ldots \ldots \ldots \ldots \ldots$

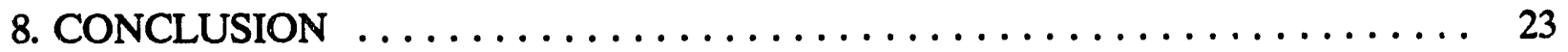

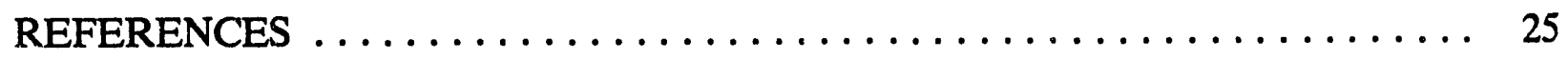

APPENDIX A - SURFACE REACTION RATE CONSTANTS $\ldots \ldots \ldots \ldots$

APPENDIX B - DETAILED ANALYTICAL SOLUTION . . . . . . . . . 31

APPENDIX C - ITER CDA DIVERTOR LOADING ESTIMATES . . . . . . 34

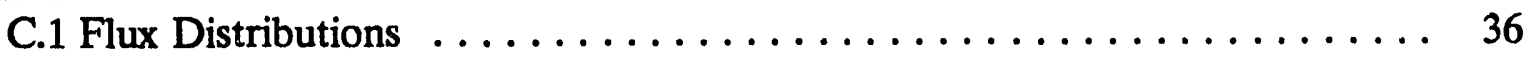

C.2 Implantation Depth $\ldots \ldots \ldots \ldots \ldots \ldots \ldots \ldots \ldots \ldots \ldots \ldots$ 


\section{INTRODUCTION}

There is a general need in the fusion reactor safety community for a means of quickly estimating the amount of tritium retained in and transmitted through plasma-facing components during fusion reactor operations. This model should contain information about the material used, the conditions under which it is operating, and the reactor design. There are fairly sophisticated codes such as TMAP4, ${ }^{1}$ that may be used for modelling transients using detailed mechanisms in one spatial dimension. In general these require considerable skill and familiarity to use and inventiveness to model complex systems such as plasmafacing components. The need exists for a more transparent model that will give results similar to those of the detailed codes, but be more readily accessible. It should be simpler and faster than full-scale time-transient codes.

To that end, I have assembled a simplified model, specifically oriented toward the Lotus $123^{\mathrm{m}}$ Version 3.0 or higher spread sheet software, to give indications of the steadystate inventory and permeation rate through plasma facing components and estimate characteristic times for reaching steady-state. The model is still one-dimensional, but complex structures or loadings may be accommodated by building up collections of onedimensional segments that may be solved simultaneously. With the spread-sheet application of this model the user specifies the number of segments and a material for the component. Pertinent properties for that material are then copied by macros into the active region of the spread sheet from data blocks developed for that purpose. These data may be adjusted to suit particular interests. The dimensions, heat and particle loading values, and heat transfer characteristics are then added by the user. Macro commands accomplish the calculation of the steady state inventory and permeation rates and time constants for building up to those values. The model may also be coded into a standard high-level language such as Fortran or $\mathrm{C}++$, though I have made no effort to do that. From here on I assume the spread-sheet format.

In keeping with the goal of making the model straightforward and relatively userfriendly, it is appropriate to employ a number of assumptions and simplifications. While this may detract slightly from the accuracy of the calculations, the uncertainty in the result due to such approximations is no greater than that in the data making up the problem definition.

-In this report I first describe the physical assumptions in the model. Then I develop the equations and boundary conditions and solution algorithm to be used in the model. I include next some comparison calculations for the present model with the TMAP4 code, for which this model is a simplification. Finally, I include some sample calculations for the International Thermonuclear Experimental Reactor (ITER) Conceptual Design Activity (CDA) divertor plate under representative operating conditions.

\section{MODEL DESCRIPTION}

The model assumes permeation through a plate, which may be considered to be any structure where the permeation is effectively one-dimensional. That means that curvature effects, complex configuration, and variations of heat and particle loadings on the surface 
are by definition inconsequential. A particle flux of both deuterium and tritium is assumed to be implanted into the material at a single fixed depth and to diffuse under the action of both concentration and thermal gradients to the surfaces. Atoms diffusing to the plasma side are assumed to undergo second-order recombination. Also on the plasma side, an assumed gas pressure, such as would be present in some divertor concepts, leads to dissociative absorption. The model assumes Baskes' recombination coefficient ${ }^{2}$ as a default, but an arbitrary value or formula may be used. At the downstream or coolant side, the reaction/release rate to the coolant is assumed to be in one of three regimes: (1) second order recombination as at the upstream surface; (2) first order in the dissolved tritium concentration such as may be the case if there were a hydrogen-receptive impurity in the coolant stream in low concentrations; and (3) a zero concentration boundary that would result from a high concentration of hydrogen-receptive complexes in the coolant stream or under circumstances of extremely fast recombination. This flexibility makes the model applicable to a wider variety of designs. Receptive complexes will doubtlessly be available in water-cooled applications and are likely to be present as impurities in inert gas coolant streams as well. In each case I assume that for tritium control purposes, the coolant stream is sufficiently free of $\mathrm{D} / \mathrm{T}$ bearing molecules that dissociative reentry into the wall is negligible.

Table 1. Material parameters stored in data blocks and used to evaluate tritium inventory and permeation rate.

$\boldsymbol{\kappa}=$ Thermal conductivity of the material $(\mathrm{W} / \mathrm{mK})$
$\epsilon=$ Thermal emissivity of the material surface $\left(\mathrm{W} / \mathrm{m}^{2} \mathrm{~K}^{4}\right)$
$S_{0}=$ Tritium solubility preexponential (atom fraction $\left./ \mathrm{Pa}^{1 / 2}\right)$
$E_{\mathrm{s}}=$ Enthalpy of solution $(\mathrm{eV})$
$D_{0}=$ Tritium diffusivity preexponential $\left(\mathrm{m}_{2} / \mathrm{s}\right)$
$E_{\mathrm{d}}=$ Diffusion activation energy $(\mathrm{eV})$
$\rho=$ Effective tritium trap density (atom fraction)
$E_{\mathrm{t}}=$ Effective tritium trap energy $(\mathrm{eV})$
$Q^{*}=$ Heat of transport or Soret coefficient $(\mathrm{eV})$
$N=$ Number density of the material atoms $\left(\mathrm{m}^{-3}\right)$

Table 1 lists the intrinsic material properties required by the model. These values are stored for 304 stainless steel, beryllium, tungsten, and pyrolytic graphite in storage blocks in the spread sheet and are retrieved by macro commands once the material has been identified. Data for additional materials may be added by the user. Table 2 lists problemspecific parameters that must be entered by the user for each application. However, these are left in the spread sheet from one computation cycle to the next, so they need not be entered each time. It may be argued that thermal emissivity, $\epsilon$, should be in Table 2 as an application dependent parameter, but it is a simple thing to change it during problem data entry, so where it is listed is of little consequence. The sticking factors, $\alpha_{i}$, are indications of the surface cleanliness. Values of unity should be used unless additional information 
Table 2. Design-specific parameters used to calculate tritium inventory and permeation rates.

$A=$ Area of the $p l a t e\left(\mathrm{~m}^{2}\right)$
$L=$ Thickness of the plate $(\mathrm{m})$
$Q=$ Thermal heat load to the surface $\left(\mathrm{W} / \mathrm{m}^{2}\right)$
$J_{i}=$ Combined $(\mathrm{D} / \mathrm{T})$ ion flux to the surface $\left(\right.$ ion $\left./ \mathrm{m}^{2} \mathrm{~s}\right)$
$\phi=$ Fraction of $J_{i}$ that is tritium
$\delta=$ Implantation depth (m)
$\alpha_{1}=$ Effective sticking coefficient at $\mathrm{plasma}$ face
$\alpha_{2}=$ Effective sticking coefficient at coolant face
$h_{c}=$ Heat transfer coefficient to coolant $\left(\mathrm{W} / \mathrm{m}^{2} \mathrm{~K}\right)$
$T_{c}=$ Temperature of the coolant stream $(\mathrm{K})$
$T_{\mathrm{h}}=$ Temperature of the plasma-side radiation sink $(\mathrm{K})$

suggests lower values.

\section{ANALYSIS}

\subsection{The Physical Model}

The terminology fir the model development that follows is illustrated in Figure 1. The plate has thickness $L$. A heat flux, $Q$, is intercepted by the plasma-side surface, which has emissivity $\epsilon$. That surface radiatively communicates with a sink at temperature $T_{\mathrm{h}}$. The coolant is at temperature $T_{\mathrm{c}}$, and heat enters the coolant from the plate with convective heat transfer coefficient $h_{c^{*}}$. The implantation flux, $J_{\mathrm{i}}$, is deposited at a shallow depth, $\delta$, which is exaggerated in the figure. The hydrogen concentration, $n(\mathrm{x})$, is assumed to be made up of a fraction, $\phi$, of tritium and (1- $\phi)$ of deuterium. Diffusivity and other parameters used are those for tritium. I assume that deuterium concentrations will be $(1-\phi) / \phi$ times those of the tritium. Note that the concentration profiles shown are non-linear. This is symbolic of the action of the Soret effect ${ }^{3}$ and temperature-dependent diffusivity. Plasma-facing components are likely to carry sufficiently heavy heat loads that thermally-driven transport must be considered. Also, because diffusivity varies with temperature, and temperature varies with distance, $x$, across the plate, the concentration profile will be generally non-linear even in the absence of the Soret effect.

On the plasma side, annotated with subscript 1 , the sticking coefficient, $\alpha_{1}$, leads to recombination coefficient for $\mathrm{T}_{2}$ molecules, $K_{r_{1}}$ (see Appendix A). The same pattern applies to the coolant side, annotated with subscript 2 . The recombination rate for DT molecules is modified for the mass difference. However, since I envision reaction of dissolved tritium with other species in addition to itself and deuterium, I use a more general term, surface reaction coefficient, $K_{\mathrm{s}}$, at the downstream surface. For the zero-concentration condition, the rate constant becomes infinitely large. The rationale for computing these rate constants is given in greater detail in Appendix A. The reaction rate constants together 


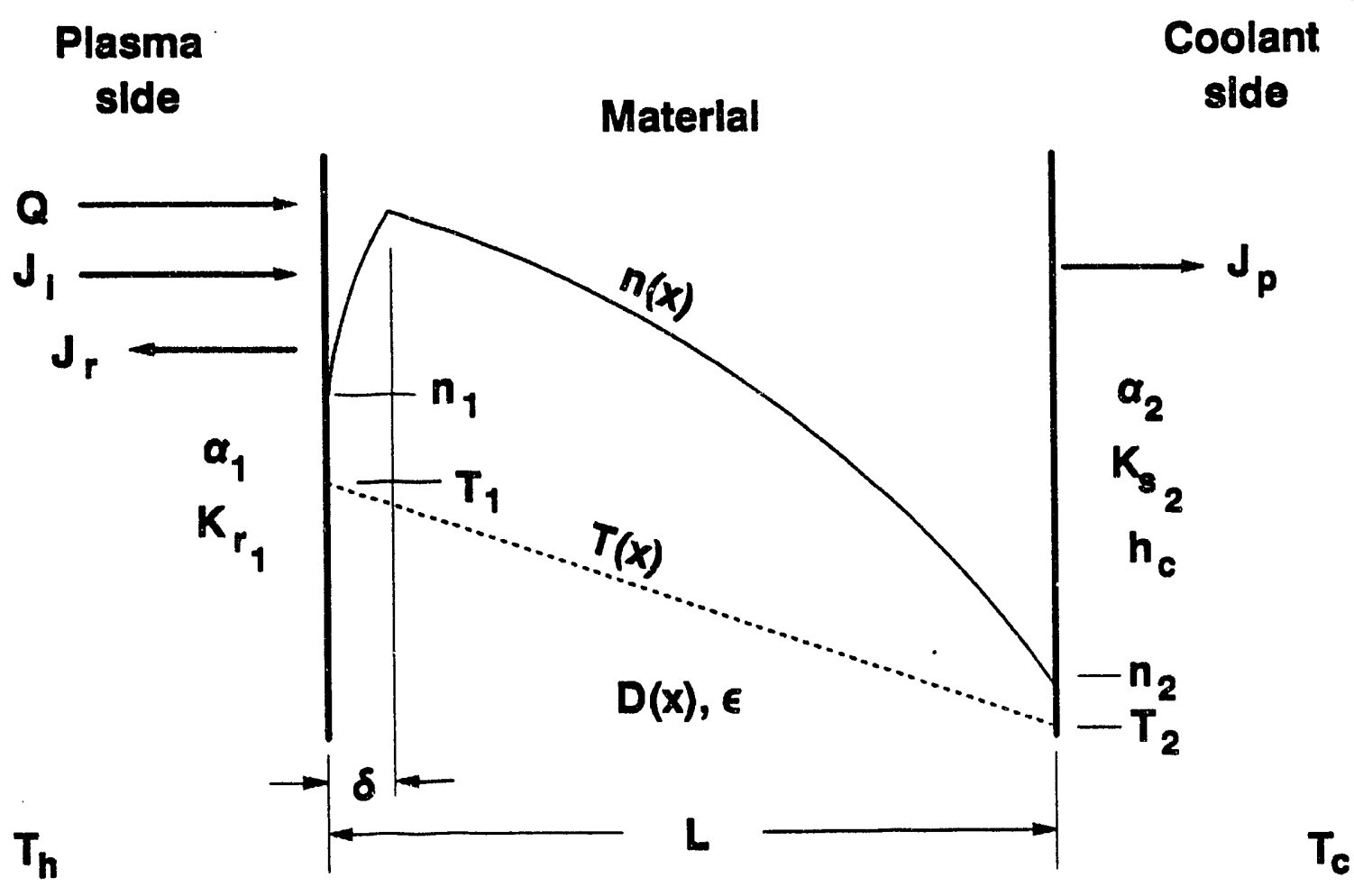

Figure 1. Notation used in developing the analytical model for inventory and permeation rates.

with values of $n$, or alternately values for $D, Q^{*}, \nabla T$ and $\nabla n$, at the surface describe a permeation flux, $J_{\mathrm{p}}$, and a reemission flux, $J_{\mathrm{r}}$, from the plate material. These fluxes are evaluated as comprising tritiated molecular rates only. Both $T_{2}$ and DT are considered specifically. $D_{2}$ rates are not included. Only tritium atoms are counted in the diffusing species concentrations and fluxes, although both $\mathrm{D}$ and $\mathrm{T}$ are assumed to be present.

\subsection{Heat Transfer}

The temperature gradient, $\nabla T$, is assumed to be constant over the thickness of the material. The upstream and downstream temperatures are calculated from simple heat transfer considerations. The lower temperature, $T_{2}$, is given by

$$
T_{2}=T_{c}+\frac{Q_{\text {eff }}}{h_{c}} \text {. }
$$

$Q_{\text {eff }}$ is the effective heat load carried by the material after radiant heat transfer from the plasma-side surface is subtracted off. Hence, $T_{1}$ would be 


$$
T_{1}=T_{2}+Q_{\text {eff }} \frac{L}{\kappa}
$$

The effective heat load is

$$
Q_{\text {eff }}=Q-\sigma \epsilon\left(T_{1}^{4}-T_{h}^{4}\right)
$$

where $\sigma$ is the Steffan-Boltzmann constant. The view factor of unity is assumed. Departures from that may be implemented by changing the value of $\epsilon$. Equations (1) to (3) are solved in the model by Newton-Raphson iterative convergence. If desired, a formula for calculating thermal conductivity, $\kappa$, as a function of mean temperature $e^{a}$ may be used in lieu of a fixed value and included in the iteration.

\subsection{Surface Reactions}

I assume that second-order recombination of $T_{2}$ molecules can be successfully modelled using Baskes' formalism with an appropriate choice of sticking coefficient, $\alpha$, and a correction to account for DT molecule formation. However, some other formula may be employed at the user's discretion. The equation for Baskes' $K_{\mathrm{r}}$ is

$$
K_{r}=\frac{\alpha \exp \left(\frac{2 E_{s}-E_{x}}{k T}\right)}{\sqrt{2 \pi M k T} S_{o}^{2} N^{2}}
$$

where $M$ is the mass of the molecule formed by the recombination, $k$ is the Boltzmann constant, and $T$ is the local temperature at the surface, which is assumed to be the same as that of molecules just above the surface. The quantity $E_{\mathrm{x}}$ is a threshold energy that an atom must surmount to enter the material. It is given by

$$
\begin{array}{ll}
E_{x}=E_{s}+E_{d}, & E_{s}+E_{D}>0 \\
E_{x}=0, & \text { Otherwise }
\end{array}
$$

Additional detail is in Appendix A.

Note that the recombination rate in Eq.(4) is quite temperature sensitive. For that reason, it is calculated at the temperature of the surface where it applies. It is also dependent on the molecular mass. In the calculations, I have used the mass of the $T_{2}$ molecule $(M=6 \mathrm{amu})$. If a change is required, the user may adjust the initial constant ${ }^{\mathrm{b}}$

${ }^{a}$ Refer to this temperature in the computational formula in the cell as TM.

${ }^{b}$ Change the constant from 1.0728E+24, the reciprocal of that part of the radical in Eq.(4) exclusive of temperature: divide it by the square root of $1 / 6$ the mass in amu of the molecule formed. 
in the cell formula for $\mathrm{K}_{\mathrm{r}}$ to suit, or the correction may be included in the all-purpose knob, $\alpha$.

At the coolant surface, the surface reaction rate constant, $K_{\mathrm{s}}$ is calculated from Eq.(4) if the process is second order. If first order, the rate constant must include the concentration of the reacting species, so the constant is calculated according to the procedures in Appendix A, and the formula or value must be manually inserted into the appropriate cell(s) of the spread sheet.

The reemission flux to the plasma side is found from

$$
J_{r}=K_{r_{1}} n_{1}^{2}\left(2+2 \sqrt{\frac{6}{5}} \frac{1-\phi}{\phi}\right) \equiv \mu K_{r_{1}} n_{1}^{2}
$$

where the term in parentheses and defined as $\mu$ accounts for DT molecule production under the assumption that deuterium concentrations are $\left(\frac{1-\phi}{\phi}\right)$ times tritium concentrations. In the same way the permeation flux to the coolant side is assumed to be found from

$$
J_{p}=\mu K_{s_{2}} n_{2}^{2}
$$

if it is second order. If first order, the flux is

$$
J_{p}=K_{s_{2}} n \text {. }
$$

Note that for first order reactions involving hydrogen isotopes, the deuterium would not be involved since the concentration, $n$, is assumed to be only tritium. If the $n=0$ boundary condition is active at the downstream face, the permeation flux is evaluated from

$$
J=-D\left(\nabla n+\frac{n Q^{*}}{k T^{2}} \nabla T\right)
$$

Once again, these fluxes and the inventories calculated refer just to the tritium.

\subsection{Diffusion}

The fundamental equation for dissolved atom flux in the presence of temperature gradient effects is found in Eq.(9) where $D$ is the diffusivity, given by 


$$
D=D_{o} \exp \left[-\frac{E_{d}}{k T}\right]
$$

and the heat of transport or Soret coefficient, $Q^{*}$, characterizes thermally driven transport. At steady state, except for depth $\delta$, mass conservation requires that $\nabla \cdot J=0$ or that

$$
\nabla D \cdot\left(\nabla n+\frac{n Q^{*}}{k T_{2}} \nabla T\right)+D\left[\nabla^{2} n+\frac{Q^{*}}{k T^{2}} \nabla n \nabla T-2 \frac{n Q *}{k T^{3}}(\nabla T)^{2}\right]=0 \text {. }
$$

Recall that $\nabla T$ is taken to be constant. There will be a discontinuity in $\nabla n$ at $\delta$ because of the injection of the tritium ion flux, $\phi J_{\mathrm{i}}$, there. Differentiating Eq.(10), substituting for $\nabla D$ in Eq.(11), and rearranging gives

$$
\nabla^{2} n+\left[\frac{E_{d}+Q *}{k T^{2}} \nabla T\right) \nabla n+\left[\frac{E_{d}-2 k T}{\left(k T^{2}\right)^{2}} Q *(\nabla T)^{2}\right] n=0 .
$$

The proper solution of this equation is very tedious and complex because the temperature used $i_{i}$ the coefficients of $n$ and its derivatives changes with $x$. The approach is summarized in Appendix B. If instead of the variable temperature, $T$, in Eq.(12) I use some average temperature, say $\langle T\rangle$, that is assumed constant, and if the energies in the coefficients of $n$ and its derivatives are constants, then Eq.(12) becomes a simple equation with constant coefficients. Specifically, let

$$
\beta=\frac{E_{d}+Q *}{2 k<T>^{2}} \nabla T
$$

and

$$
\gamma=\frac{E_{d}-2 k<T>}{\left(k<T>^{2}\right)^{2}} Q *(\nabla T)^{2} .
$$

Then the indicial equation for Eq.(12) is

$$
D^{2}+2 \beta D+\gamma=0
$$

for which the roots are 


$$
\lambda_{1}=-\beta+\sqrt{\beta^{2}-\gamma}
$$

and

$$
\lambda_{2}=-\beta-\sqrt{\beta^{2}-\gamma} .
$$

Then the distribution of $n$ over $x$ will be simply

$$
n=C_{1} \exp \left(\lambda_{1} x\right)+C_{2} \exp \left(\lambda_{2} x\right)
$$

where $C_{1}$ and $C_{2}$ are constants that depend on the boundary conditions. Because the boundary conditions will be different on $0 \leq x \leq \delta$ than on $\delta<x \leq \mathrm{L}$, I will use $\hat{C}_{1}$ and $\hat{C}_{2}$ to refer to the constants on $0 \leq x \leq \delta$.

If $\gamma=0$, as it will if $Q^{*}=0$ or $\nabla T=0$ or $E_{\mathrm{d}}=2 k T$, then the solution becomes

$$
n=C_{1} \exp (-2 \beta x)+C_{2} \text {. }
$$

The assumptions that $\nabla T \neq 0$ and $E_{\mathrm{d}} \neq 2 k T$ for problems of interest with this model certainly seem reasonable. If I adopt the restriction that $Q^{*} \neq 0$, though it may be very small, then I avoid the encumberment of the second solution, that given by Eq.(19).

The question of how to select the best value for $\langle T\rangle$ arises. If I use the highest temperature available, $T_{1}$, the magnitudes of the $\lambda_{\mathrm{i}}$ will be their lowest, and depending on the algebraic sign of the $\lambda_{i}$, the estimates of $n$ will have the furthest depriture above or below the "true" value. Conversely, if the lowest value, $T_{2}$, is used ro compute the $\lambda_{\mathrm{i}}$, then the opposite extreme will hold. A better value for $\langle T\rangle$ will lie between $T_{1}$ and $T_{2}$. Because plasma-facing components will be of high thermal conductivity by design, the difference between $T_{1}$ and $T_{2}$ should be small compared with $\langle T\rangle$, so the uncertainty of the computed $n(x)$ should not be large. One could simply use the arithmetic mean temperature, but a slightly better one may be found by observing that the generalized forcing function for thermal gradient effects is really $-\nabla\left(Q^{*} / k T\right)$ which for constant $Q^{*}$ becomes ${ }^{4} Q^{*} / k T^{2} \nabla T$. Hence, for constant $Q^{*}$ and $\nabla T$, the average contribution to the driving force for mass flow ought to be given by the coefficients evaluated at the temperature for which $1 / T^{2}$ is at its average value

$$
\frac{1}{\langle T\rangle^{2}}=\frac{1}{L} \int_{0}^{L} \frac{1}{T^{2}} d x=\frac{1}{T_{1} T_{2}} \text {. }
$$

\section{Therefore,}




$$
<T>=\sqrt{T_{1} T_{2}}
$$

should give a slightly better solution.

\subsection{Boundary Conditions}

The solution must be evaluated over two intervals, one to the plasma side and one to the coolant side of the $x=\delta$ surface. To evaluate the constants, $\hat{C}_{1}, \hat{C}_{2}, \mathrm{C}_{1}$, and $\mathrm{C}_{2}$, note that at the plasma side $(x=0)$,

$$
\mu K_{r_{1}} n_{1}^{2}=D_{1}\left(\left.\nabla n\right|_{x=0}+\frac{n_{1} Q^{*}}{k<T>^{2}} \nabla T\right)
$$

where $D_{1}$ is the diffusivity evaluated at $t_{4}=0$. For convenience, define

$$
\eta \equiv \frac{Q^{*}}{k<T>^{2}} \nabla T \text {. }
$$

Then, when the functional forms of $n$ and $\nabla n$ from Eq.(18) are put into Eq.(22) and $x \rightarrow 0$, I get

$$
\frac{\mu K_{r_{1}}}{D_{1}}\left(\hat{C}_{1}+\hat{C}_{2}\right)^{2}-\hat{C}_{1}\left(\lambda_{1}+\eta\right)-\hat{C}_{2}\left(\lambda_{2}+\eta\right)=0
$$

At the coolant side $(x=\mathrm{L})$, either $n_{2}=0$ or

$$
\mu^{(m-1)} K_{s_{2}} n_{2}^{m}=-D_{2}\left(\left.\nabla n\right|_{x=L}+n_{2} \eta\right)
$$

where $\mathrm{m}=1$ or 2 depending on the circumstances. Performing similar operations at this boundary gives either

$$
C_{1} \exp \left(\lambda_{1} L\right)+C_{2} \exp \left(\lambda_{2} L\right)=0
$$

for the $n_{2}=0$ case or for $m=1$ or 2

$$
\begin{aligned}
\frac{\mu^{(m-1)} K_{s_{2}}}{D_{2}}\left[C_{1} \exp \left(\lambda_{1} L\right)+C_{2} \exp \left(\lambda_{2} L\right)\right]^{m}+C_{1} \exp \left(\lambda_{1} L\right)\left(\lambda_{1}+\eta\right) \\
+C_{2} \exp \left(\lambda_{2} L\right)\left(\lambda_{2}+\eta\right)=0 .
\end{aligned}
$$


At $x=\delta$ the diffusive fluxes away from the $x=\delta$ surface must difference to equal the implantation flux:

$$
D_{\delta}\left(\left.\nabla n\right|_{x=\delta^{-}}-\left.\nabla n\right|_{x=\delta^{*}}\right)=\phi J_{i}
$$

Here, evaluations at $\delta^{*}$ and $\delta^{+}$refer to the plasma side and the coolant side, respectively, of the surface at $x=\delta$. This gives

$$
\frac{\phi J_{i}}{D_{\delta}}+\left(C_{1}-\hat{C}_{1}\right) \lambda_{1} \exp \left(\lambda_{1} \delta\right)+\left(C_{2}-\hat{C}_{2}\right) \lambda_{2} \exp \left(\lambda_{2} \delta\right)=0
$$

Finally, at $x=\delta$, the values of $n$ for the two segments of the solution must be equal,

$$
\left.n\right|_{x=\delta^{-}}=\left.n\right|_{x \rightarrow \delta^{*}} \text {. }
$$

That leads to

$$
\left(C_{1}-\hat{C}_{1}\right) \exp \left(\lambda_{1} \delta\right)+\left(C_{2}-\hat{C}_{2}\right) \exp \left(\lambda_{2} \delta\right)=0
$$

Equations (24), (26) or (27), (29), and (31) constitute a sufficient set to solve for the constants $\hat{C}_{1}, \hat{C}_{2}, C_{1}$, and $C_{2}$. Note that Eqs.(24) and (27) in the $m=2$ case are non-linear in general whereas Eqs.(26), (29) and (31) are linear in the constants to be solved for.

\subsection{Solution Algorithm}

The method of solution chosen for the general case when $n_{2} \neq 0$ is to estimate a value for $\hat{C}_{1}$ and use Eq.(24) to determine $\hat{C}_{2}$. That equation may be rewritten in explicit form as

$$
\hat{C}_{2}=\frac{D_{1}\left(\lambda_{2}+\eta\right)}{2 \mu K_{r_{1}}}\left[1 \pm \sqrt{\hat{c}_{1} \frac{\left(\lambda_{1}-\lambda_{2}\right)}{\left(\lambda_{2}+\eta\right)^{2}} \frac{4 \mu K_{r_{1}}}{D_{1}}+1}\right]-\hat{C}_{1} .
$$

If this is to have a real solution, then

$$
\hat{C}_{1} \geq-\frac{\left(\lambda_{2}+\eta\right)^{2} D_{1}}{4 \mu K_{r_{1}}\left(\lambda_{1}-\lambda_{2}\right)}
$$

This condition may be accommodated by using $\hat{C}_{1}=1$ as the initial guess for $\hat{C}_{1}$. A further requirement is that $\hat{C}_{1}+\hat{C}_{2} \geq 0$ for a positive $n_{1}$. Since each of the terms in the right side of Eq.(33) is positive definite, $\hat{C}_{1}$ may be negative and still give a real solution. However, if the $\left(\lambda_{2}+\eta\right)$ term in Eq.(32) is negative or if it is positive and $\hat{C}_{1}$ is also positive, then only the positive-sign solution will give a positive $n_{1}$. If $\hat{C}_{1}$ is negative but satisfies Eq.(33) 
and $\left(\lambda_{2}+\eta\right)$ is positive, then there is the potential for a double-valued solution. However, since a double-valued solution is non-physical, I discard the positive-sign solution when the negative-sign solution is available. ${ }^{c}$

Once $\hat{C}_{2}$ is found from Eq.(32), the next step is to combine Eqs.(29) and (31) to get

$$
C_{2}=\hat{C}_{2}-\frac{\phi J_{i}}{D_{\delta}\left(\lambda_{2}-\lambda_{1}\right)} \exp \left(-\lambda_{2} \delta\right)
$$

where $\mathrm{D}_{\delta}$ is just $\mathrm{D}$ evaluated at $T(\delta)$. This gives $C_{2}$ directly from $\hat{C}_{2}$.

With $C_{2}$ thus determined, $C_{1}$ may be found from Eq.(27). This may be a non-linear equation, and the Newton-Raphson method is used to find the zero. The initial guess is taken as unity.

Finally, with estimates of $C_{1}, C_{2}$, and $\hat{C}_{2}$, a better value of $\hat{C}_{1}$ is found from Eq.(31). The process is repeated until the correction to $\hat{C}_{1}$ becomes acceptably small.

If the $n_{2}=0$ condition applies, then Eq.(26) simplifies Eq.(27) even further, but does not require a departure from the general solution algorithm.

\section{INVENTORY AND PERMEATION}

Having thus determined the constants for the tritium concentration equations, the inventory is a trivial integral of the concentration, $n$, over the thickness of the plate,

$$
\begin{array}{r}
I=A\left\{\frac{\hat{C}_{1}}{\lambda_{1}}\left[\exp \left(\lambda_{1} \delta\right)-1\right]+\frac{\hat{C}_{2}}{\lambda_{2}}\left[\exp \left(\lambda_{2} \delta\right)-1\right]+\frac{C_{1}}{\lambda_{1}}\left[\exp \left(\lambda_{1} L\right)-\exp \left(\lambda_{1} \delta\right)\right]\right. \\
\left.+\frac{C_{2}}{\lambda_{2}}\left[\exp \left(\lambda_{2} L\right)-\exp \left(\lambda_{2} \delta\right)\right]\right\} .
\end{array}
$$

The total steady-state mobile atom inventory is simply the sum of inventories in the several individual plate segments. Permeation current for each of the assumptions on $m$ is calculated from the basic transport equation, Eq.(9):

$$
\Gamma_{p}=-D_{2}\left[C_{1} \exp \left(\lambda_{1} L\right)\left(\lambda_{1}+\eta\right)+C_{2} \exp \left(\lambda_{2} L\right)\left(\lambda_{2}+\eta\right)\right] A
$$

The total steady-state permeation current is the simple sum of the currents from each segment.

${ }^{c}$ I also found that convergence requires that practice. 


\subsection{Transient Times}

The time evolution of the permeation current, $\Gamma_{\mathrm{p}}$, in the classical one-dimensional problem where a plate with concentrations $n(x, t)$ where $n(x>0,0)=0$ and $n(0,0)=n_{\mathrm{o}}$ and where $n(L, t)=0$, is given by the equation

$$
\Gamma_{p}=\frac{n_{o} D A}{L}\left\{1+2 \sum_{m=1}^{\infty}\left[(-1)^{m} \exp \left[-m^{2} \frac{t}{\tau_{D}}\right)\right]\right\}
$$

where the characteristic diffusion time, $\tau_{\mathrm{D}}$, is given by

$$
\tau_{D}=\frac{L^{2}}{\pi^{2} D}
$$

This is represented graphically in Figure 2.

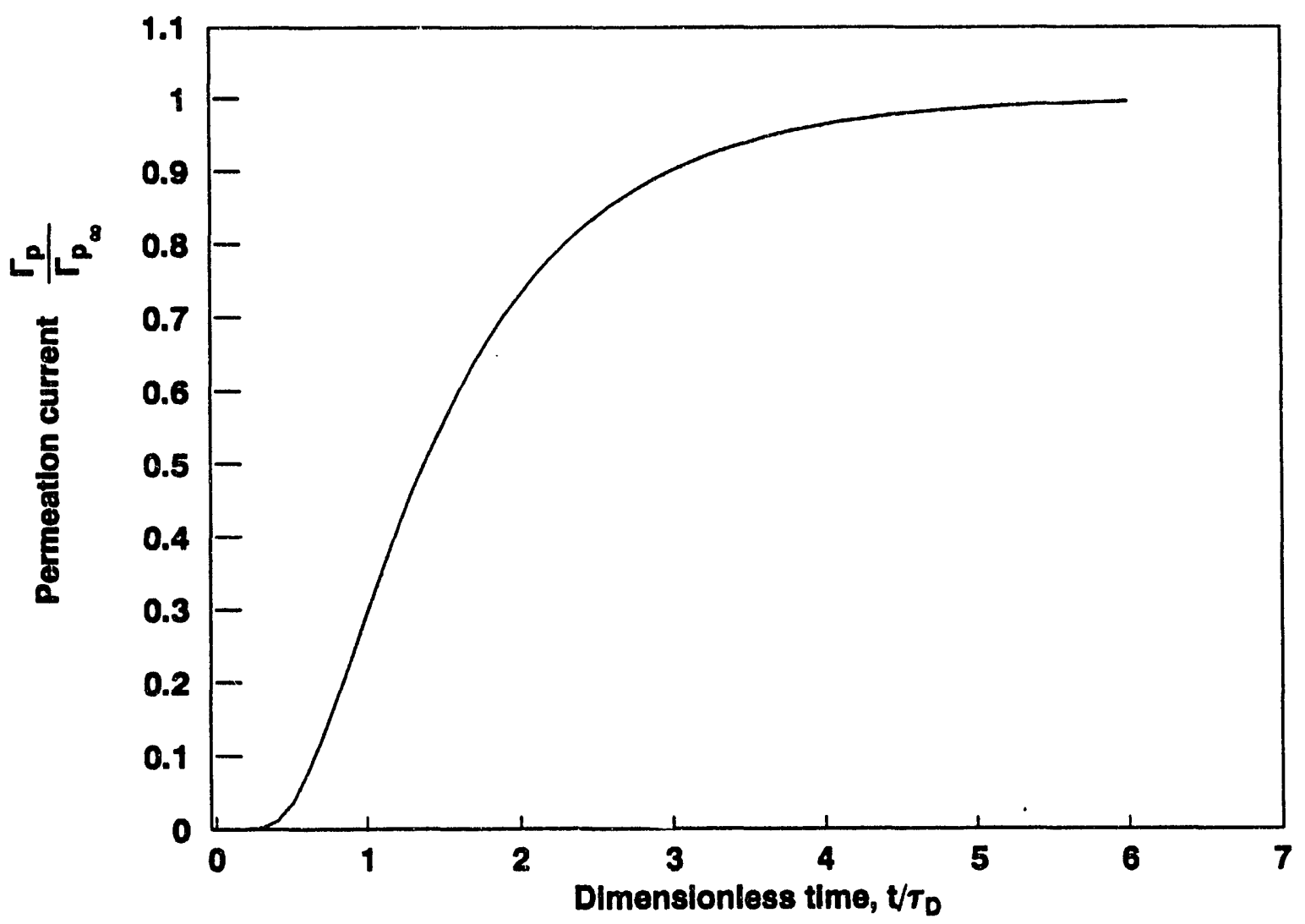

Figure 2. Permeation transient history for the classical problem of diffusion through a plate. 
The permiation rate development is estimated for the above idealized problem as essentially a saturating exponential function with time constant $\tau_{D}$ and offset from the $t=$ 0 axis by $r_{D} / 2$. Inventory buildup has the same time constant but no delay. If different segments have different diffusion times, then the inventory of each segment will grow at a different rate, scaled by the diffusion time, $r_{D}$.

\subsection{Trapping}

This idealized model assumes that no trapping or surface effects influence the steadystate permeation. In plasma-facing components, however, both these effects may be expected. Detailed discussion of the effects of trapping on permeation transients is provided elsewhere, ${ }^{5}$ but I give a brief summary here. Two limits must be considered. In the effective diffusivity limit, the traps are present and delay the permeation transient, but their effects are virtually indistinguishable from a reduction in the diffusivity. The effective diffusivity is given by

$$
D_{\text {eff }}=\frac{D}{\sum_{j} \frac{1}{\zeta_{j}}}
$$

where the trapping parameter, $\zeta_{\mathrm{j}}$, is

$$
\zeta_{j}=\frac{\lambda^{2} \nu}{\rho_{j} D} \exp \left(-\frac{E_{t_{j}}}{k T}\right) \text {. }
$$

In this equation $\lambda$ is the material lattice spacing, $\nu$ is the Debye frequency (typically $10^{13} \mathrm{~s}^{-1}$ ), and $\rho_{j}$ and $E_{t_{j} j}$ are the trap concentration fraction and trapping energy for the $\mathrm{jth}$ trap. When the trapping characteristics are known, Eqs.(39) and (40) may be used to find $D_{\text {eff }}$ which is then substituted for $D$ in Eq.(38) to find the characteristic permeation time, $\tau_{\mathrm{D}}$. Then breakthrough and permeation transient development may be estimated for PFC components using Figure 2. The inventory in the effective diffusivity limit is just $1+1 / 5$ times the inventory given by the classical diffusion model.

The other limit is the strong trapping limit. There, the traps so dominate atom movement that effectively none of the diffusing tritium can get past them until they become filled. Hence, a front moves through the plate behind which traps are all filled and the mobile concentration is non-zero and ahead of which neither trapped nor mobile atoms of the diffusing species are present. In that case the breakthrough time is given by

$$
\tau_{b}=\frac{L^{2} \rho N}{2 n_{\delta} D} \text {. }
$$

In the strong trapping limit, no permeation occurs until $\tau_{b}$, and then permeation quickly jumps to its steady state value. The total inventory in this limit is $1+\rho N / n$ times the mobile atom inventory. 
The determination as to whether the effective-diffusivity or the strong-trapping limit may apply lies in the comparison of $\zeta$ and $n / \rho N$. If $\zeta \gg n / \rho N$ then the effective diffusivity limit applies and vice versa. For situations when $\zeta \approx n / \rho N$, neither of these limits is satisfactory, and an intermediate value must be found. As an interpolating function, I find the value of breakthrough time in each of the limits and make an interpolation for the estimated breakthrough time, $\tau_{\text {esl }}$, based on the relative values of the ratio, $n / 5 \rho N$ according to

$$
\tau_{e s t}=\tau_{b}-\frac{\tau_{b}-\tau_{D}}{1+\left(\frac{n}{\zeta \rho N}\right)^{r}}
$$

where $\mathrm{r}$ is a power to be determined by comparison with more accurate calculations. This relationship, together with the influence of $r$, is shown in Figure 3.

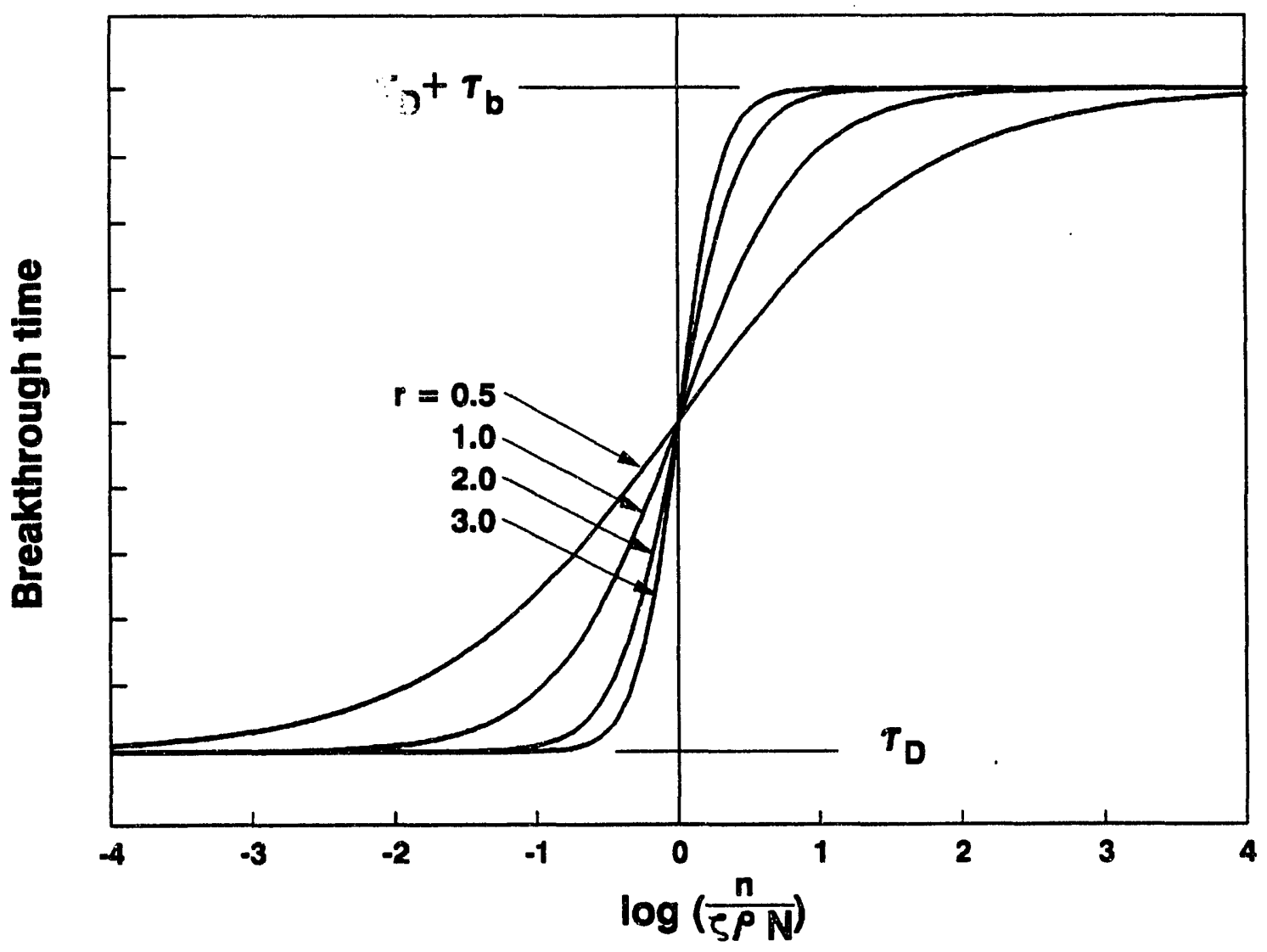

Figure 3. Transition of $\tau_{\text {eff }}$ from $\tau_{\mathrm{d}}$ to $\tau_{\mathrm{b}}$ with the trapping discriminant, $n / \zeta \rho N$. 


\subsection{Surface Effects}

Surface effects act to change the concentrations of the mobile species at the surfaces and consequently throughout the thickness of the plate. Plasma-side surface effects act principally to alter $n_{1}$ and the maximum $\mathrm{D} / \mathrm{T}$ concentration, $n_{\delta}$. Because these values are established relatively quickly in the transient, these surface effects have little effect on breakthrough time in either limit. Coolant-side surface effects alter the value of $n_{2}$ thus changing the overall inventory and the shape of the permeation transient once breakthrough has occurred, but there is little effect on the time at which permeation begins since that will always be limited by diffusion. Surface effects of both kinds have been included in the modelling presented above, so they have already been taken into account for their effects on inventory.

\section{USAGE}

The model is designed to run mostly automatically, but with great flexibility. It exists as a .WK3 file, suitable for running with Lotus $123^{m}$ release 3.0 or higher. When the file is retrieved into Lotus $123^{\mathrm{m}}$, the problem preparation macro (labelled 10 ) automatically begins. It accomplishes several functions. One is the establishment of a number of range definitions in column B, which is the column in which the basic problem is stored. It also copies information about the previous execution of the problem to a range labelled STORAGE for future reference if needed. This may be information that was stored with the file or used in a previous execution of the loaded file. It also erases all data from columns to the right of column B.

Next, an input screen is presented where the user is asked to provide the number of segments to be included in the present problem, the order, $\mathrm{m}$, of the reaction at the coolantside surface, and the identity of the material these segments are made of. Up to 52 segments may be defined. ${ }^{d}$ The list of available material names for property parameters is given. One is selected by moving the highlight to the name of the material desired and pressing [Enter]. Note that only one material may be specified at that point in the input, but material property values may be manually altered before completing the calculation. Error trapping routines prevent the entering of other than numbers for the first two entries and a non-label entry for the material name. Entering any other material name than the ones listed will result in problem failure. ${ }^{e}$

\footnotetext{
${ }^{\mathrm{d}}$ This limit is not a firm one and may be adjusted by modifying the macro to clear out a larger area before replicating the material data, subject to machine memory limitations. Different materials may be accommodated by manually inserting data for the other materials or copying material data from the PROPLIST range.

eAdditional materials may be added before restarting the problem by (1) adding the name to the material list in the screen (get there with [F5] opnscrn), (2) defining a range (enter /rnc< rangename> and specify the block size), having the same name as the entry in the screen, that will contain the material property values (not the material name), and (3) entering values for material properties. A convenient area for storing this range may be found adjacent to the
} 
After these data are entered, the macro replicates the material properties and the equations for calculating permeation, inventory, and transient times into as many columns as needed for the number of segments specified by the user beginning in column $B$. Numbers in row 1 indicate the segment number. Problem definition data are copied from the storage area into rows 2 to 13 . These data may fill fewer columns than used in the current problem. If so these data or other comparable data will need to be copied or entered into the unfilled active columns. The macro stops to allow the user to make any changes, additions, or alterations needed in the problem definition data, the material properties data, or even in the equations. Remember that only columns with segment numbers in row 1 are active and that data and formulas in each of those columns must be fully defined. Changes made to the formulas at that point, recombination coefficients for example, must be copied to all active columns. Problem definition data outside the active range have no effect on the problem execution.

When the problem definition is complete, the macro is restarted by entering $[\mathrm{Alt}]+[\mathbf{G}]$. At that point the ranges defined in column $\mathrm{B}$ by the initialization routine are extended to include all active columns, and the calculational algorithm is initiated. First temperatures $T_{1}$ and $T_{2}$ are found, then the constants for the concentration distribution function are determined. This is an iterative procedure and may take some time. The time required obviously depends on the speed of the computer and the number of segments used in the problem. It also depends on the problem stiffness. When convergence $e^{\text {f,g }}$ has been achieved, the viewing window is relocated to the region where the results have been calculated, beginning at the left side with segment 1 and the combined inventory. Concentration profile data are calculated and are listed below the summary data. The first six of these profiles may be viewed by entering /gv (or /gnuconcprofile if the user has. defined other graphics).

The problem may be reinitialized by entering $[\mathrm{Alt}]+[\mathbf{F 3}] \mid \mathbf{0}$. To restart the problem after calculations have been initiated with the $[\mathbf{A l t}]+[\mathbf{G}]$ and then stopped with $[\mathbf{C t r l}]+\left[\mathbf{B r}_{\mathrm{a}} \mathbf{k}\right]$, [Esc], simply enter $[\mathbf{A l t}]+[\mathbf{R}]$. The continuation point will be iteration on the concentration coefficients, $\hat{C}_{1}, \hat{C}_{2}, C_{1}$, and $C_{2}$. Temperature calculations will not be repeated by this process.

Modifications to material property data used may be made before reinitializing the problem by accessing the property list table ([F5]proplist) and making changes in the table.

existing data fields. This is accessed with [F5]proplist.

${ }^{\text {f }}$ The convergence criterion may be changed by entering [F5]conval to get to the value currently in use and entering a new value.

The row to watch is the one called C1-hat correction (row 64). It should rapidly approach the Convergence value in row 68 . If it is going too slowly, stop computation with $[\mathrm{Ctrl}]+[$ Break], [Esc] and move to row 56 ( 1 1-hat estimate). Manually change that value and restart calculations with $[\mathrm{Alt}]+[\mathrm{R}]$. Continue this process as needed to accelerate convergence. While computations are stopped, the output based on the current estimates of the coefficients may be seen by entering $[\mathrm{Alt}]+[\mathrm{F}]$. 
Those changes will be made permanent by saving the file, otherwise they will be lost when the program is exited.

\section{COMPARISON CALCULATIONS}

For a comparison with TMAP4 calculations, I have selected two problems. The first is a plate of SS-304, $2 \mathrm{~mm}$ thick and $1 \mathrm{~m}^{2}$ in area. The heat flux was $1 \mathrm{MW} / \mathrm{m}^{2}$, and the particle flux was $1 \times 10^{22} \mathrm{ion} / \mathrm{m}^{2} \mathrm{~s}$, half each of $\mathrm{D}$ and $T$. I used Baskes' recombination coefficient and assumed traps of atom fraction $1 \times 10^{-5}$ at $0.8-\mathrm{eV}$ trap energy. The same assumptions were made for the second case, except the material was changed to tungsten with a trap atom fraction of $7 \times 10^{-5}$ and a trap energy of $1.53 \mathrm{eV}$, and the heat load was increased to $5 \mathrm{MW} / \mathrm{m}^{2}$. Other parameters are listed in Table 3.

Table 3. Problem parameters used for comparison with TMAP4 for the SS-304 and tungsten plates.

A Segment area $\left(\mathrm{m}^{2}\right)$

$L$ Segment thickness (m)

Q Heat load $\left(\mathrm{W} / \mathrm{m}^{2}\right)$

Ji Implantation Flux $\left(D+T / \mathrm{m}^{2} \mathrm{~s}\right)$

Phi Tritium Fraction

delta Implantation depth (m)

Alpha-1

Alpha-2

T-c Coolant temperature (K)

T-h Radiant heat sink temp (K)

hc Convective coeff $\left(\mathrm{W} / \mathrm{m}^{2} \mathrm{~K}\right)$

MATERIAL

$\mathrm{Kh}$ Thermal conductivity (W/mK)

So Tritium solubility $\left(\mathrm{Pa}-\frac{1}{2}\right)$

Es Solution enthalpy (eV)

Do Diffusivity pre-exp $\left(\mathrm{m}^{2} / \mathrm{s}\right)$

Ed Diffusion Act Energy (eV)

$Q^{*}$ Heat of Transport (eV)

N Material number density (m-)

Rho Trap conc (atom fraction)

Et Trap energy (eV)

eps Thermal emissivity

$r$ Exponent in time estimate
1
1
0.002

1000000

$1.00 E+22$

0.5

$1.50 \mathrm{E}-08$

1

373

400

5000

SS-304

0.000012

0.144

3.70E-07

0.49

$-0.58800$

$7.46 E+28$

0.00001

0.8

0.2

0.7

$$
\begin{array}{r}
2 \\
1 \\
0.002 \\
5000000 \\
1.00 \mathrm{E}+22 \\
0.5 \\
1.50 \mathrm{E}-08 \\
1 \\
1 \\
373 \\
400 \\
5000 \\
\\
\text { Tungsten } \\
163 \\
0.000028 \\
1.04 \\
4.10 \mathrm{E}-07 \\
0.39 \\
0.2 \\
6.30 \mathrm{E}+28 \\
0.00007 \\
1.53 \\
0.15
\end{array}
$$

The permeation transient I calculated for the SS-304 with TMAP4 is shown in Figure 4 while the inventory buildup history is shown in Figure 5. Note that breakthrough took place after about 0.07 days, and the shape of the curve is essentially like that in Figure 2 indicating that this is in the effective-diffusivity regime. 


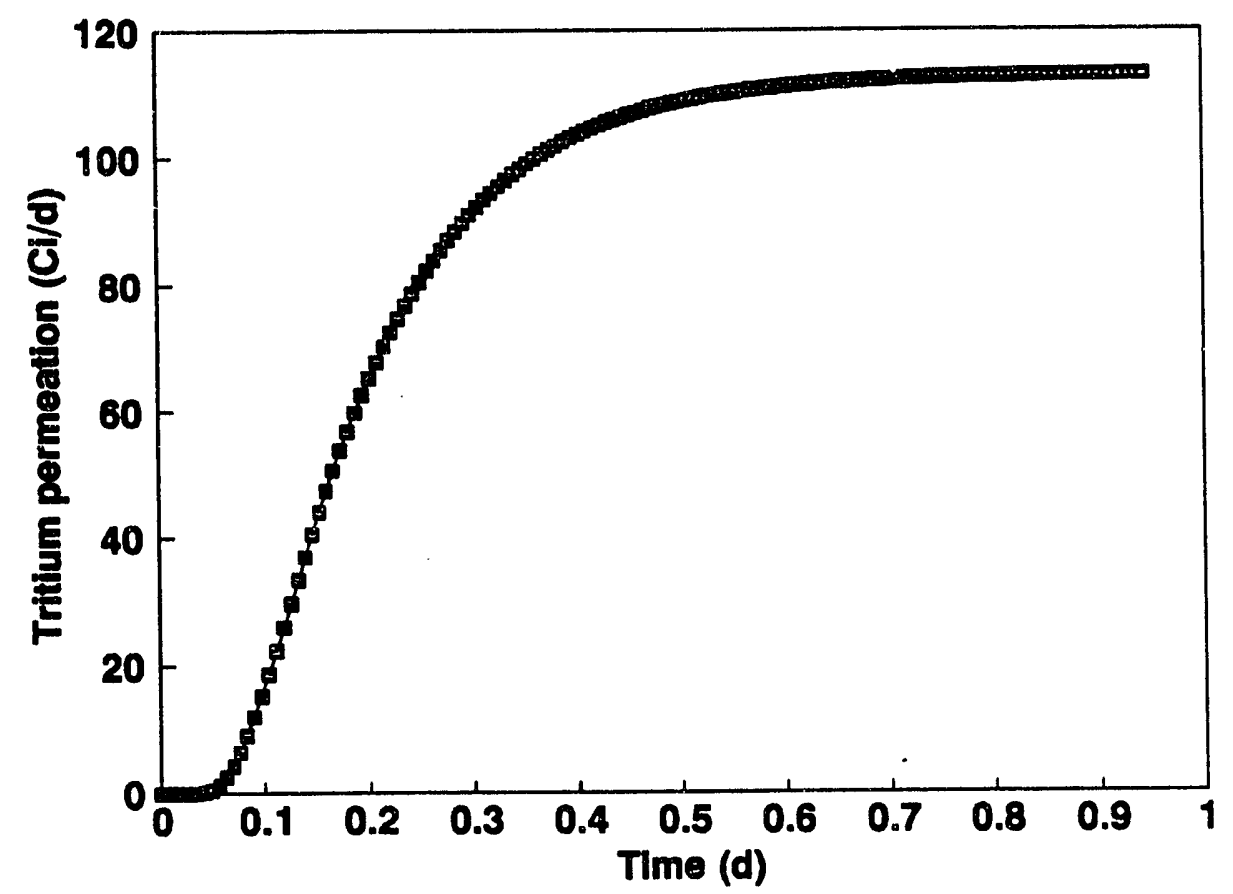

Figure 4. Permeation transient calculated by TMAP4 for the first compari-son problem.

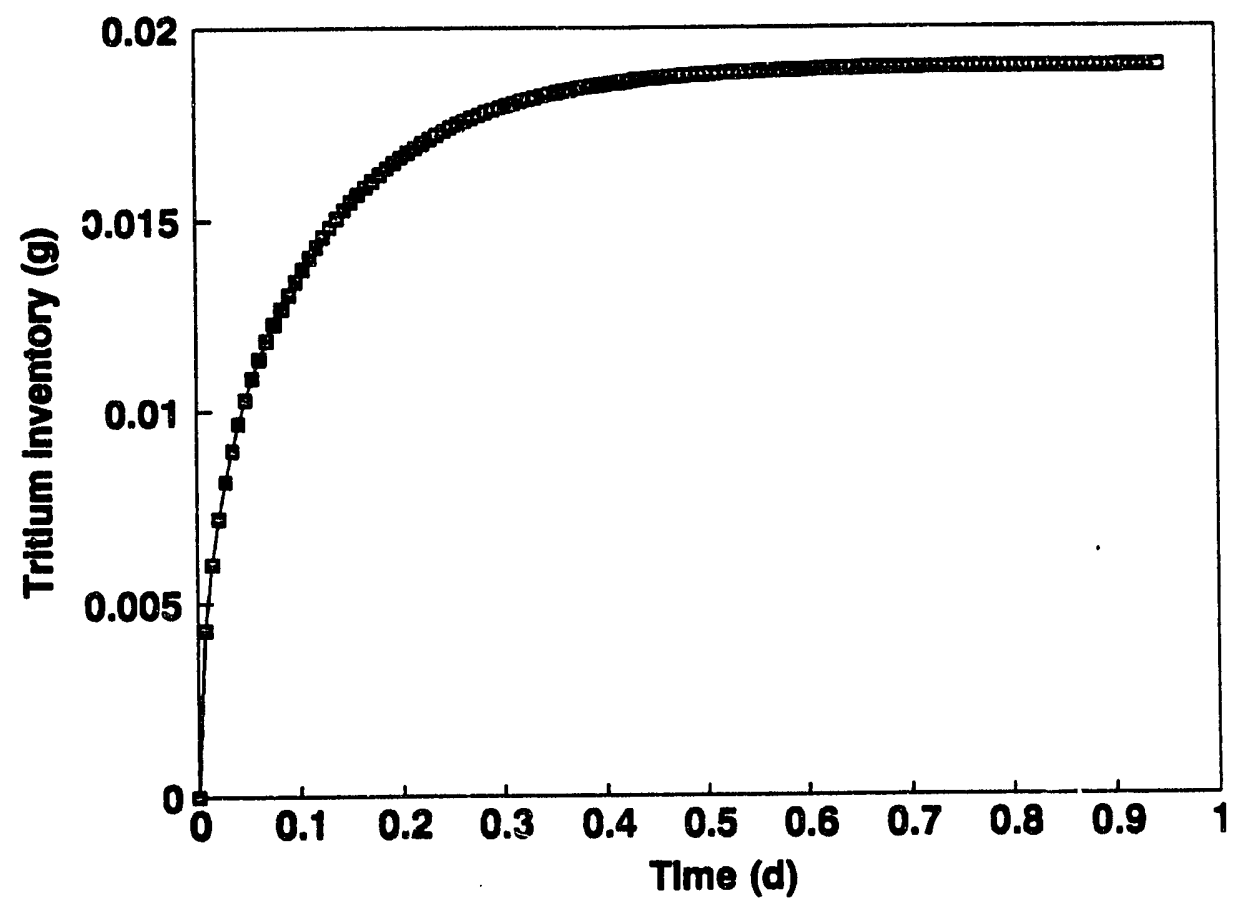

Figure 5. Inventory buildup for the first comparison problem. 
The present spreadsheet model using the same material properties and loadings found that the steady-state inventory in the plate was $0.0189 \mathrm{~g}$, the permeation was $113 \mathrm{Ci} / \mathrm{day}$, and the breakthrough time was 0.068 days using $r=0.7$. All three values are very close to those shown in the figures. Concentration profiles calculated by the present spread-sheet program are compared with those generated by TMAP4 in Figure 6. Agreement is quite good although there is some concavity in the TMAP4 calculations that does not appear in the present model profile.

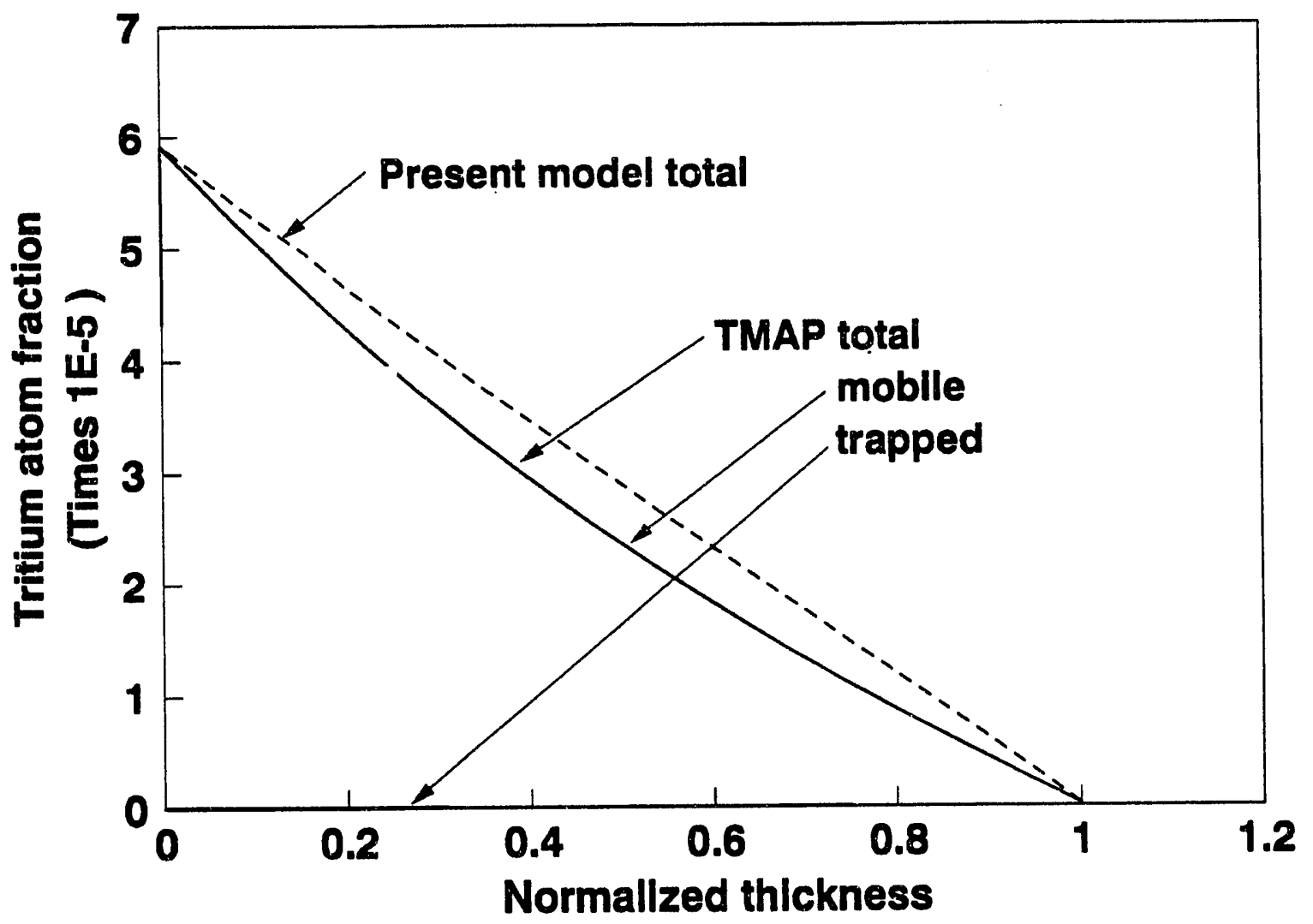

Figure 6. Comparison of steady-state total inventory calculated by the present spreadsheet model with TMAP4 calculations for a 2-mm SS-304 plate.

For the tungsten case, the value of $\mathrm{Q}^{*}$ has not yet been measured, but I believe it will be near that of niobium, which has been measured. ${ }^{6}$ Trapping effects we have observed experimentally are characterized by a $1.5-\mathrm{eV}$ trap energy and a concentration of $7 \times 10^{-5}$ atom fraction. Again I use a $1-\mathrm{m}^{2}$ plate area. Permeation transient and total (trapped plus mobile) inventory data calculated are given in Figure 7 and Figure 8. For comparison, the present spread-sheet based model predicts a breakthrough time of 0.00474 days or 6.82 minutes when the estimating exponent, $r$, is 0.7. It appears from Figure 7 that the breakthrough time calculated by TMAP4 is much smaller. The diffusion breakthrough time in the absence of trapping is 0.33 minutes. That appears very close to the intercept of the steepest slope in the figure. That value requires $r$ to be larger than 2. The current model predicts a permeation rate of $1,033 \mathrm{Ci} /$ day which is only $73 \%$ of the $1417 \mathrm{Ci} /$ day predicted 


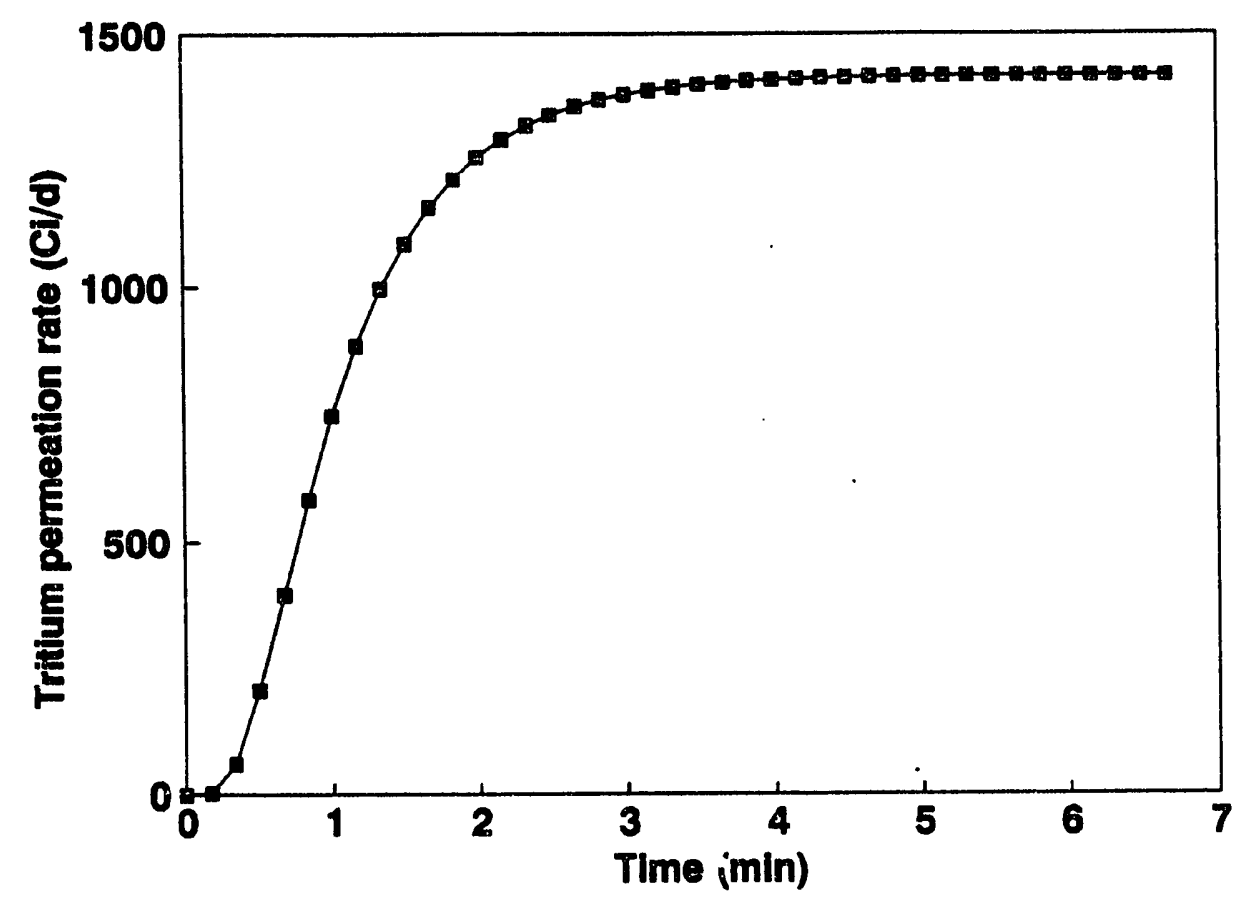

Figure 7. Permeation transient calculated by TMAP4 for the 2-mm tungsten plate.

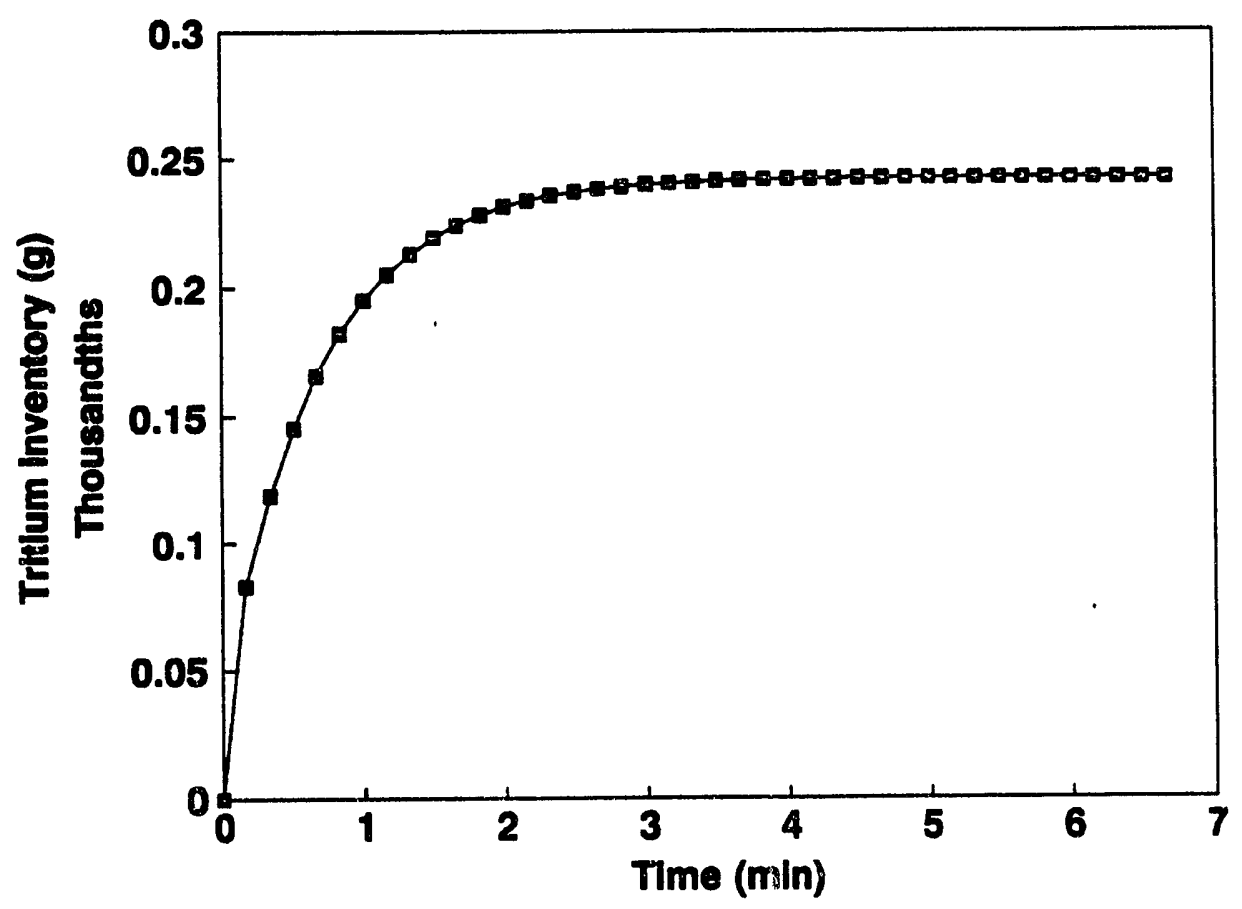

Figure 8. Tritium inventory buildup in tungsten plate as calculated by TMAP4. 
by TMAP4. The total inventory predicted by the spread-sheet model is $0.248 \mathrm{mg}$, inexcellent agreement with TMAP4 results in Figure 8.

Figure 9 shows the concentration profiles calculated by TMAP4 and by the present model for the tungsten case. The agreement on total tritium atom concentration is quite good. Note that the TMAP4 results show the trapped-to-mobile ratio varying from about 0.5 at the $\mathrm{x}=0$ side to more than 0.7 at $\mathrm{x}=\mathrm{L}$. The present model gives 0.59 .

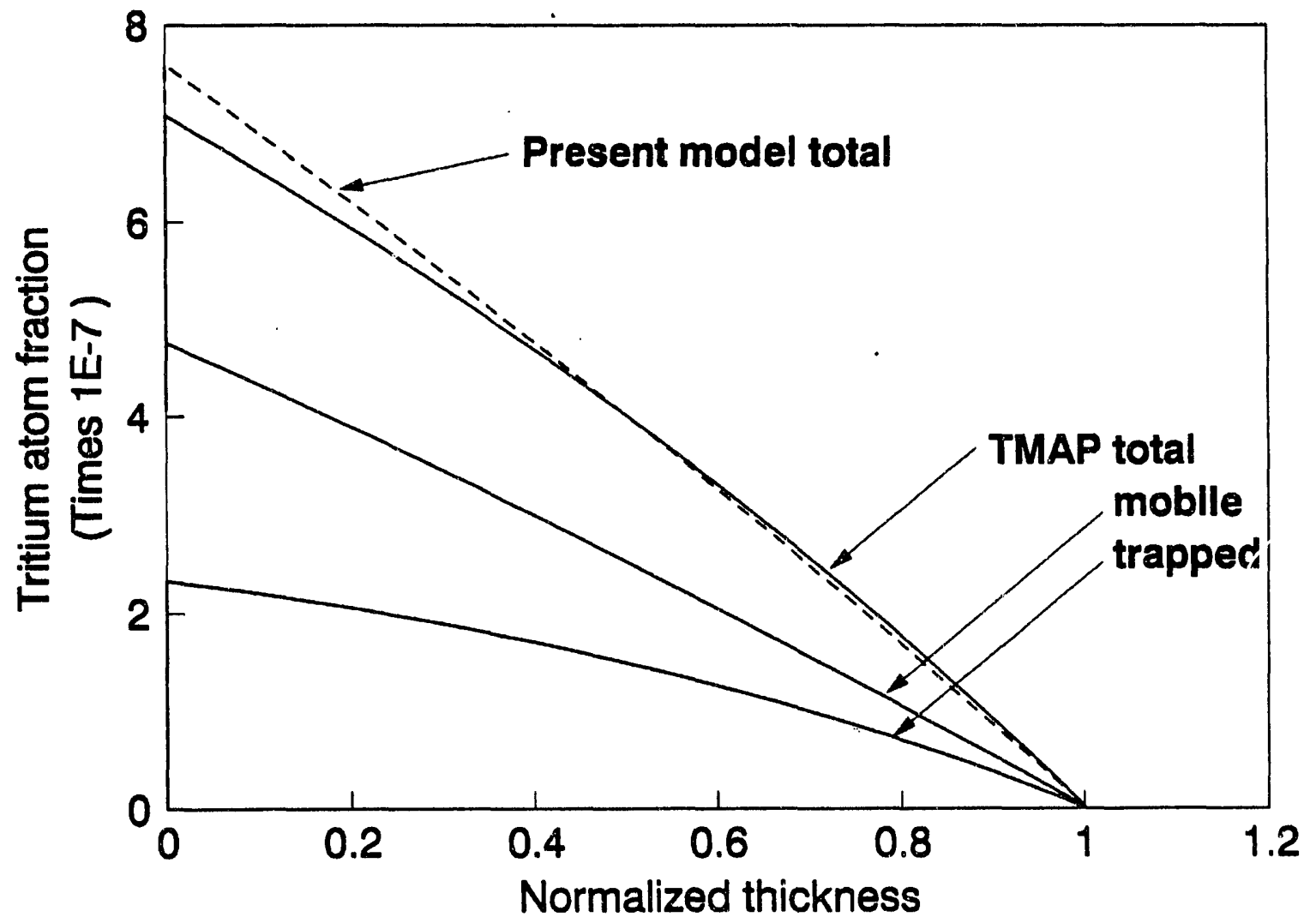

Figure 9. Concentration profiles calculated for the tungsten plate by TMAP4 and by the present model.

\section{ITER DIVERTOR CALCULATIONS}

The design for the ITER divertor is, for now, fluid at best. However, as a further demonstration of the ability of this model, I give here a calculational result using the ITER Conceptual Design Activity (CDA) divertor design as an example. ${ }^{7}$ The CDA design called for a double null poloidal divertor system with divertor plates at the top and bottom. The baseline design called for the divertor plate to be surfaced with a carbon composite for the Physics Phase of operations but tungsten and beryllium were considered as backups. For the higher loadings of the Technology Phase, the surface was planned to be tungsten or beryllium. The base plate and front plate were to be directly cooled by pressurized water, nominally at $50-65^{\circ} \mathrm{C}^{8}$ 
Several authors have presented analyses of heat and particle loadings to the outer divertor plate, for example Harrison and Hotston, ${ }^{9}$ but corresponding loadings for the inboard plates are frequently lacking. In Appendix C I show how I used available information to extrapolate to particle and heat flux density distributions over both inboard and outboard divertor plates. I consider here only a Technology Phase divertor using beryllium as the plasma-facing material in the analyses I present here. The divertor is divided into eighteen annular segments. Areas, average heat flux and particle flux densities, and estimated plasma temperatures are given for each of these segments in Appendix C, Table C-2. I used $2 \mathrm{~mm}$ for the plate thickness, $373 \mathrm{~K}$ for the coolant temperature, $400 \mathrm{~K}$ for the radiant heat sink temperature, and $5000 \mathrm{~W} / \mathrm{m}^{2} \mathrm{~K}$ for a convective coefficient throughout. Material properties used for beryllium are shown in Table 4. Note that the value of $\mathrm{Q}^{*}$ is an estimate based on values found in the literature for other materials. ${ }^{10}$

Table 4. Material properties assumed for beryllium.

$\begin{array}{lr}\text { Kh Thermal conductivity }(\mathrm{W} / \mathrm{mK}) & 95 \\ \text { So Tritium solubility }\left(\mathrm{Pa}-\frac{1}{2}\right) & 0.0572 \\ \text { Es Solution enthalpy (eV) } & 1 \\ \text { Do Diffusivity pre-exp }\left(\mathrm{m}^{2} / \mathrm{s}\right) & 8.00 \mathrm{E}-09 \\ \text { Ed Diffusion Act Energy }(\mathrm{eV}) & 0.363 \\ Q^{\star} \text { Heat of Transport }(\mathrm{eV}) & 0.103 \\ N \text { Material number density }\left(\mathrm{m}^{-3}\right) & 1.25 E+29 \\ \text { Rho Trap conc (atom fraction) } & 0.01 \\ \text { Et Trap energy (eV) } & 0 \\ \text { eps Thermal emissivity } & 0.15 \\ r \text { Exponent in time estimate } & 0.7\end{array}$

The total steady-state tritium inventory calculated by this model was 4,917 grams. Atom fraction profiles for segments 1.6 are given in Figure 10. They are representative of other segments as well. Note that except for segment 1 where there was effectively no temperature gradient, the concentration profiles show an unusual shape. This is a consequence of the temperature sensitivity of the tritium diffusion coefficient and of the Soret effect. The higher concentration values in segment 1 are because its cooler temperature makes recombination more difficult, so higher concentrations are required to achieve surface fluxes needed to balance the implantation flux.

The tritium permeation rate in steady state was calculated to be $74,182 \mathrm{Ci} /$ day. Most of that comes from the segments at the strike points. The rate at which that value is approached is shown in Figure 11. The curve in that figure is the total of contributions from all of the segments and represents the permeation flux after the start of steady-state operation.

That is also essentially the rate at which the inventory builds in the divertor, except the inventory would have no initial lag time. When the plasma is removed, the release of tritium from the divertor would not go so quickly unless the divertor temperature were 


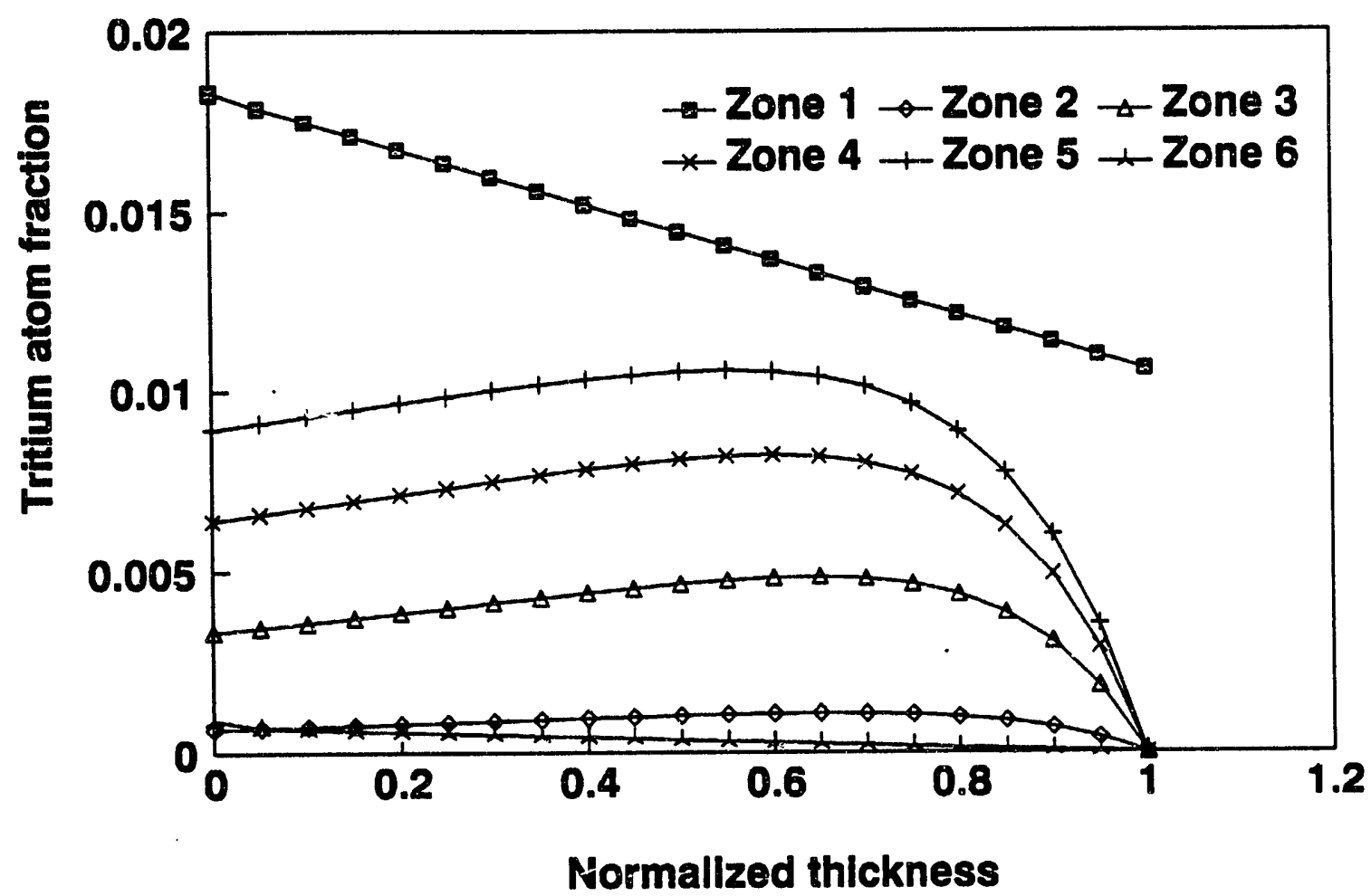

Figure 10. Tritium concentration profiles calculated for a 2-mm thick beryllium surface to the ITER divertor in the Technology Phase.

maintained at least as high. I should point out that the model predicted temperatures of greater than $2,000 \mathrm{~K}$ at the strike points. Those temperatures are too high for beryllium operation, and it obviously requires an extrapolation of transport parameters. Rather large contributions to the overall inventory are calculated for the large, low-heat-flux segments away from the strike points. This is indicated by the concentration profile in segment 1 shown in Figure 10. Not only is the tritium atom fraction higher in steady state, but the areas are also very large. However, for these components, the breakthrough time is on the order of 20 days, so it would take a long time to build in (40-day time constant). It would also take a long time to empty.

\section{CONCLUSION}

The model presented here is a simplification of more detailed models that simulate various aspects of tritium permeation and retention in plasma-facing structures. It has been developed to take advantage of the flexibility offered by spreadsheet programs such as Lotus $123^{\mathrm{m}}$, however, it may also be recast into the environment of Fortran-77 or other high-level computer languages. 


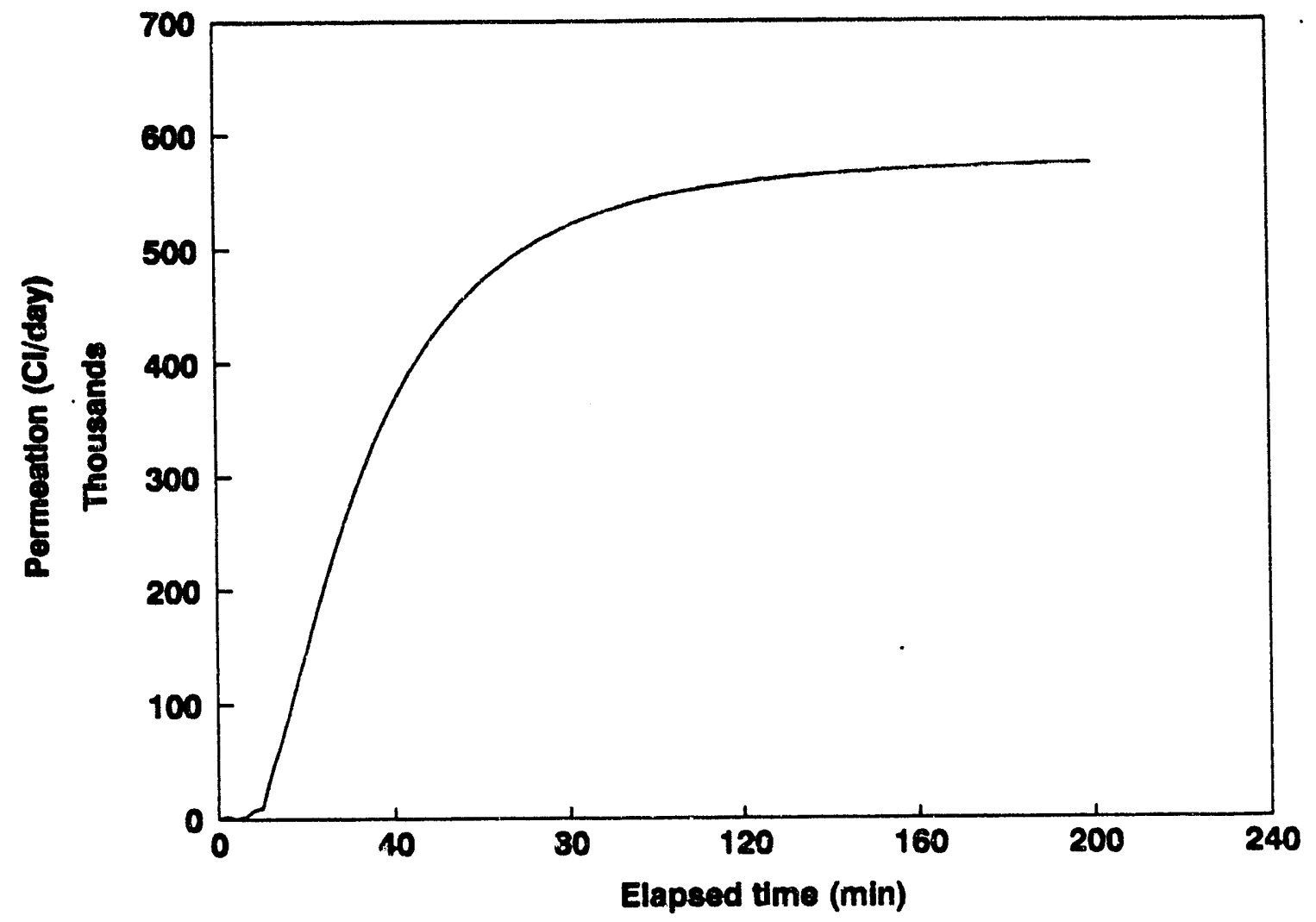

Figure 11. Tritium permeation from the divertor plate to the coolant stream.

The model operates by solving for the steady-state solution for a given set of input parameters. Transient histories are then estimated by determining in what regime the transient may be expected to fall and applying the appropriate normalized transient curve. Results have been compared with similar calculations using TMAP4 and found to be essentially the same.

The model is intended mainly for parametric analyses involving tritium permeation and retention in various plasma-facing structures. While usage in it present form requires a reasonable familiarity with the Lotus $123^{\mathrm{m}}$ spreadsheet program, it takes advantage of built-in automatic functions and commands to facilitate efficient use, even by a novice. 


\section{REFERENCES}

1. G. R. Longhurst, D. F. Holland, J. L. Jones, and B. J. Merrill, "TMAP4 User's Manual," EGG-FSP-10315, June 12, 1992; see also D. F. Holland and B. J. Merrill, "Analysis of Tritium Migration and Deposition in Fusion Reactor Systems," Proceedings of the 9th Symposium on Engineering Problems of Fusion Research, Chicago, Mlinois, October 26-29, 1981, pp, 1209-1212.

2. M. I. Baskes, "A Calculation of the Surface Recombination Rate Constant for Hydrogen Isotopes on Metals," Journal of Nuclear Materials 92, (1980) p. 318.

3. G. R. Longhurst, "The Soret Effect and its Implications for Fusion Reactors," Journal of Nuclear Materials 131, (1985) pp. 61-69.

4. See for instance, H. B. Callan, Thermodynamics and Introduction to Thermostatistics, John Wiley \& Sons, Inc., New York, 1985, Ch. 14.

5. G. R. Longhurst, "Trapping Effects on Diffusion Transients," EGG-FSP-9789, July 1991, Idaho National Engineering Laboratory, Idaho Falls, Idaho.

6. M. Sugisaki, H. Furuya, and S. Mukai, "Temperature Dependence of $\mathrm{Q}^{*}$ tor Protium, Deuterium and Tritium in Niobium," Journal of the Less-Common Metals, 107 (1985) pp. 79-88.

7. T. Kuroda et al., "ITER Plasma Facing Components," ITER Documentation Series, No. 30, International Atomic Energy Agency, Vienna, 1991.

8. T. Kuroda et al., Ibid., Table VII.4.1-3.

9. M. F. A. Harrison and E. S. Hotston, "Edge Plasma and Surface Conditions in ITER," Journal of Nuclear Materials 176 \& 177 (1990) 256-261.

10. M. Sugisaki, H. Furuya, H. Sekiya, and K. Hashizume, "Thermal Diffusion of Tritium and Protium in Alpha Phase of Zirconium." Fusion Technology 14 (Sep. 1988) pp. 723-728. 


\section{APPENDIX A - SURFACE REACTION RATE CONSTANTS}

A hydrogen isotope atom at the surface of a material must form into a molecule with some other atom or complex before it will thermally desorb from the surface under normal circumstances. That reaction may be described by a chemical equation:

$$
m H+n Y=H_{m} Y_{n}
$$

in which $\mathrm{m}$ atoms of hydrogen isotope combine with $\mathrm{n}$ atoms of reactive complex, $\mathrm{Y}$, to form the $H_{m} Y_{n}$ molecule. That reaction is characterized by forward and backward rate constants, the ratio of which is the equilibrium constant for the reaction. Several models have been developed for evaluating these constants. I will enumerate two.

Baskes' model ${ }^{\mathrm{A} 1}$ assumes that the flux of molecules from the gas state to the dissolved atom state is given by the product of the molecule arrival rate, the probability that the molecule will dissociate and stay on the surface, and the probability that the atoms will then enter into the solution state rather than recombine and return to the gas state which is taken as the reference for energy considerations. This is the backward process in Eq.(A-1). The backward reaction rate is thus

$$
R_{b}=\frac{P \alpha}{\sqrt{2 \pi M k T}} \exp \left(-\frac{E_{x}}{k T}\right)
$$

Here the $\alpha$ is the probability of remaining on the surface (the sticking factor) assuming no activation energy required for dissociation, and the Boltzmann (exponential) factor is the probability that the atom will enter into the dissolved state. The characteristic energy for that factor, $E_{\mathrm{x}}$, is the energy of the potential barrier that separates the solution state from the free gas state. The Baskes model assumes $E_{\mathrm{x}}=E_{\mathrm{s}}+E_{\mathrm{d}}$ (no additional energy required at the surface) if that quantity is positive and zero otherwise.

The forward process, recombination, is assumed to proceed as

$$
R_{f}=K_{r} n_{1}^{2}
$$

Now it is assumed that the rate constant, $K_{\mathrm{r}}$ that applies at equilibrium applies whether equilibrium is established or not. In equilibrium, assuming Sieverts' law means

$$
n_{1}=S_{o} \exp \left(-\frac{E_{s}}{k T}\right) \sqrt{P}
$$

Combining Eqs.(A-3) and (A-4) allows solution for $K_{\mathrm{r}}$ as 


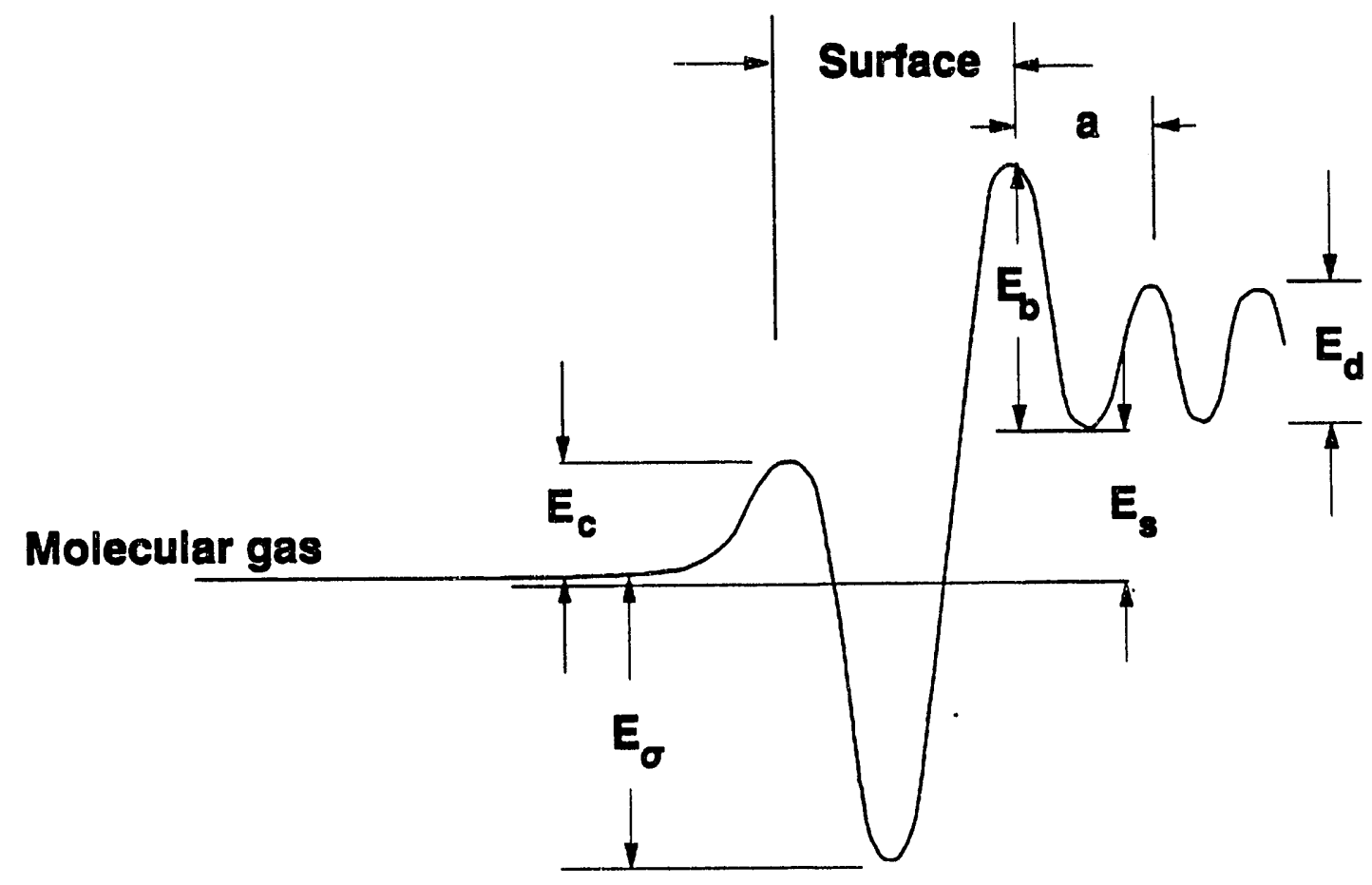

Figure A-1. Chemical potential distributions in the vicinity of the surface as assumed by Pick and Sonnenberg.

$$
K_{r}=\frac{\alpha}{\sqrt{2 \pi M k T} S_{o}^{2}} \exp \left(\frac{2 E_{s}-E_{x}}{k T}\right)
$$

Another model, after Pick and SonnenbergA2 assumes chemical potential distributions in and near the surface such as shown in Figure A-1. There $E_{c}$ is a surface barrier potential or dissociation activation energy that must be surmounted by each of the two atoms before the molecule will dissociate and the atoms become bound to the surface. $E_{\sigma}$ is the chemisorption potential for atoms at the surface. $E_{\mathrm{s}}$ is the enthalpy of solution, $E_{\mathrm{d}}$ is the diffusion activation energy, and $E_{\mathrm{b}}$ is a potential for emergence of dissolved atoms to the surface.

Assume that the flux of atoms to the surface is given by 


$$
F_{1}=\frac{2 P}{\sqrt{2 \pi M k T}}(1-\theta)^{2} \alpha_{o} \exp \left(-\frac{2 E_{c}}{k T}\right)
$$

where $\theta$ is the fractional surface coverage of chemisorbed hydrogen atoms, $\alpha_{0}$ is effectively the same sticking factor as $\alpha$ in the previous development. The factor $(1-\theta) \exp \left(-\frac{E_{c}}{k T}\right)$ is raised to the second power because a vacant surface site must be found for each of the dissociation products and each must get past the surface barrier potential.

Atoms recombine, leave the surface, and reenter the gas phase at a rate

$$
F_{2}=2 \nu \exp \left[-\frac{2\left(E_{c}-E_{\sigma}\right)}{k T}\right] \theta^{2} N^{4 / 3}
$$

where $\nu$ is again the Debye frequency $\left(10^{13} \mathrm{~s}^{-1}\right), N$ is the metal atom spatial density, and $E_{\sigma}$ is negative. The flux of atoms at the surface into the bulk is

$$
F_{3}=\theta N^{2 / 3} \nu \exp \left(-\frac{E_{s}+E_{b}-E_{\sigma}}{k T}\right)
$$

while the rate at which they return from the bulk is

$$
F_{4}=n_{1} a(1-\theta) \nu \exp \left(-\frac{E_{b}}{k T}\right)
$$

where the lattice parameter, $a$, is equal to $N^{-1 / 3}$. The factor, $(1-\theta)$, is present because each atom moving to the surface must find an empty surface site.

The sum of these four fluxes to the surface will equal the flux of surface atoms through the material by diffusion in steady state. If diffusion is negligible, then they must sum to zero in which case both $F_{1}=F_{2}$ and $F_{3}=F_{4}$, which leads to

$$
n_{1}=\left[N^{2 / 3} \sqrt{\frac{\alpha_{o}}{\nu \sqrt{2 \pi M k T}}}\right] \exp \left(-\frac{E_{s}}{k T}\right) \sqrt{P}
$$

This is in the form of a Sieverts' law expression if the terms in brackets represent the preexponential, $S_{o}$, in the solubility formula.

The recombination coefficient is defined as 


$$
K_{r}=\frac{F_{2}}{2 n_{1}^{2}}
$$

By using Eq.(A-7) for $F_{2}$ with the supplementary relation

$$
\theta^{2}=N^{-2 / 3} \frac{\alpha_{o}}{\sqrt{2 \pi M k T}} \exp \left(-\frac{2 E_{o}}{k T}\right) P,
$$

derived from the $F_{1}=F_{2}$ and $F_{3}=F_{4}$ conditions, and substituting $S_{\mathrm{o}}^{2} \exp \left(-2 E_{\mathrm{s}} / \mathrm{kT}\right) n_{1}{ }^{2}$ for $P$, the recombination coefficient from this approach is

$$
K_{r}=\frac{\alpha_{o}}{\sqrt{2 \pi M k T} S_{o}^{2}} \exp \left(-2 \frac{E_{s}-E_{c}}{k T}\right)
$$

This is essentially the same as Baskes' model except the potential $E_{\mathrm{x}}$ in the Baskes model is replaced with $2 E_{\mathrm{c}}$. Recall that in the Baskes approach, $E_{\mathrm{x}}$ was the potential that atoms had to cross to get into the material while the $E_{\mathrm{c}}$ here is the corresponding potential required to get to the surface. The difference merely reflects the difference in the assumptions regarding the importance of surface concentrations relative to subsurface ones.

Now in the case where the dissolved hydrogen reacts with a fluid concentration of another species, the sarne general approach may be followed, but it will be far better to use an empirical value for $K_{s}$. For a cursory theoretical treatment following a similar formalism, I assume

$$
F_{1}=\frac{P}{\sqrt{2 \pi M^{\prime} k T}}(1-\theta) \alpha_{o} \exp \left(-\frac{E_{c}^{\prime}}{k T}\right)
$$

Where $M^{\prime}$ is the molecular mass of the tritium-containing molecule. Note that only the hydrogen atom is assumed required to find residence sites at the surface, and the dissociation energy, $E_{\mathrm{c}}^{\prime}$ will be different than for dissociation of a homogeneous hydrogen molecule. For the returning flux,

$$
F_{2}=\nu \theta \theta^{\prime} N^{2 \beta} \exp \left(-\frac{E_{c}^{\prime}-E_{\sigma}}{k T}\right)
$$

$\theta^{\prime}$ is the fractional coverage of the associating complex at the surface, which is assumed to be independently controlled. 
The flux to the subsurface is the same as in Eq.(A-8), and the return from the bulk is identical with that in Eq.(A-9). Again noting that at equilibrium with negligible diffusion $F_{1}=F_{2}$ and $F_{3}=F_{4}$, it is straightforward to show

$$
n_{1}=\frac{\alpha_{o} \theta^{\prime}}{\nu a \sqrt{2 \pi M^{\prime} k T}} \exp \left(-\frac{E_{s}}{k T}\right) P^{\prime}
$$

In this equation the leading coefficient serves as an equivalent Henry's law solubility preexponential for tritium solution in consequence of a pressure, $\mathrm{P}$, of the reactant from the tritium combination at the surface. Further, the same process yields from Eq.(A-9)

$$
F_{1} \equiv J_{r}=\frac{\theta^{\prime} \nu \exp \left[\frac{E_{c}-E_{\sigma}}{k T}\right)}{\frac{N^{1 / 3}}{a} \exp \left(\frac{E_{\sigma}-E_{s}}{k T}\right)+N^{-2 / 3} n_{1}} n_{1}
$$

The coefficient of $n_{1}$ is a reaction rate coefficient but it involves $n_{1}$, indicating a non-linear system. Further definition will be left to the user.

\section{APPENDIX A REFERENCES}

A1 M. I. Baskes, "A Calculation of the Surface Recombination Rate Constant for Hydrogen Isotopes on Metals," Journal of Nuclear Materials 92, (1980) p. 318.

A2 M. A. Pick and K. Sonnenberg, "A Model for Atomic Hydrogen-Metal Interactions -- Application to Recycling, Recombination, and Permeation," Journal of Nuclear Materials 131 (1985) 208-220. 


\section{APPENDIX B - DETAILED ANALYTICAL SOLUTION}

The detailed analytical solution of the present problem is made somewhat difficult by the complication of the Soret term in the transport equation. Here, I will not give the complete solution, but rather show the approach and take it far enough to make some points about its nature.

The general expression for transport is

$$
\frac{\partial n}{\partial t}=-D\left\{\nabla^{2} n+\left[\left(E_{d}+Q *\right)\left(\frac{\nabla T}{k T^{2}}\right)\right] \nabla n+\left[\left(E_{d}-2 k T\right) Q *\left(\frac{\nabla T}{k T^{2}}\right)^{2}\right] n\right\}
$$

The steady state solution is just this expression with the time derivative set to zero and the diffusivity divided out. With $n=n(x, t)$, it is straightforward to see that for any particular location in $x$, the left side of Eq.(B-1) is a function of time only, and the right side is only a function of $x$, so both sides must be equal to a constant, say $-\sigma^{2}$. The reason for the choice of negative sign is to give a bounded solution in time. I then apply the separation of variables approach and define

$$
n(x, t)=X(x) \theta(t)
$$

Putting this back into the first equation and dividing by $X \Theta$ results in

$$
\frac{\Theta^{\prime}}{\Theta}=-D\left\{\frac{X^{\prime \prime}}{X}+\left[\left(E_{d}+Q *\right)\left(\frac{\nabla T}{k T^{2}}\right)\right] \frac{X^{\prime}}{X}+\left[\left(E_{d}-2 k T\right) Q *\left(\frac{\nabla T}{k T^{2}}\right)^{2}\right]\right\}=-\sigma^{2}
$$

For a second-order equation such as this, I can without loss of generality assume that the constant of integration in the time function, $\theta(t)$, is unity, resulting in

$$
\theta=\exp \left(-\sigma^{2} t\right)
$$

For the spatial function, I will multiply through by $T^{4} / D$ and rearrange to get

$$
T^{4} X^{\prime \prime}+T^{2}\left[\left(E_{d}+Q^{*}\right)\left(\frac{\nabla T}{k}\right)\right] X^{\prime}+\left[\left(E_{d}-2 k T\right) Q *\left(\frac{\nabla T}{k}\right)^{2}-\frac{\sigma^{2}}{D}\right] X=0
$$

My first approach for this non-linear equation is to use a Maclaurin's series expansion for $X:$

$$
X=\sum_{i=0}^{\infty} X_{0}^{(i)} \frac{x^{i}}{i !}
$$

where $X_{\mathrm{o}}^{(\mathrm{i})}$ denotes the ith derivative of the function, $X$, evaluated at $x=0$. Differentiating, 


$$
X^{\prime}=\sum_{i=1}^{\infty} \frac{X_{o}^{(i)} x^{i-1}}{(i-1) !}
$$

and

$$
X^{n}=\sum_{i=2}^{\infty} \frac{X^{(i)} x^{i-2}}{(x-2) !}
$$

I note that $T=T_{1}+x \nabla T$ and make substitution of Eqs.(B-6) through (B-8) into (B-5), (B-9) to get

$$
\begin{aligned}
\left(T_{1}+x \nabla T\right)^{4} \sum_{i=2}^{\infty} \frac{X_{o}^{(i)} x^{i-2}}{(i-2) !}+\left(T_{1}+x \nabla T\right)^{2}\left[\left(E_{d}+Q *\right)\left[\frac{\nabla T}{k}\right)\right] \sum_{i=1}^{\infty} \frac{X_{o}^{(i)} x^{i-1}}{(x-1) !} \\
+\left[\left(E_{d}-2 k T\right) Q *\left(\frac{\nabla T}{k}\right]^{2}-\frac{\sigma^{2}}{D_{1}}\right] \sum_{i=0}^{\infty} \frac{X_{o}^{(i)} x^{i}}{i !}=0
\end{aligned}
$$

The next step is to carry out the implied multiplications and collect coefficients on powers of $x$. For this equation to be an identity, each coefficient must independently be zero. That leads to an infinite set of equations, one for each power of $x$ in the series including the zero power. The first three such equations are

$$
\begin{aligned}
& T_{1}^{4} \frac{X_{o}^{\prime \prime}}{0 !}+T_{1}^{2} \frac{X_{o}^{\prime}}{0 !}+\left[\left(E_{d}-2 k T_{1}\right) Q *\left(\frac{\nabla T}{k}\right)^{2}-\frac{\sigma^{2}}{D_{1}}\right] \frac{X_{o}}{0 !}=0 \\
& T_{1}^{4} \frac{X_{o}^{\prime \prime \prime}}{1 !}+4 T_{1}^{3} \nabla T \frac{X_{o}^{\prime \prime}}{0 !}+\left[\left(E_{d}-2 k T_{1}\right) Q *\left(\frac{\nabla T}{k}\right)^{2}-\frac{\sigma^{2}}{D_{1}}\right] \frac{X_{o}^{\prime}}{1 !}+2 \frac{(\nabla T)^{3}}{k} Q * \frac{X_{o}}{0 !}=0
\end{aligned}
$$

and

$$
\begin{aligned}
T_{1}^{4} \frac{X_{o}^{i v}}{2 !}+ & \left(\frac{4 T_{1}^{3} \nabla T}{1 !}+\frac{T_{1}^{2}}{2 !}\right) X^{\prime \prime \prime}+ \\
& {\left[\frac{6 T_{1}^{2}(\nabla T)^{2}}{0 !}+\frac{2 T_{1} \nabla T}{1 !}+\right.} \\
& \left.\left(\frac{E_{d}-2 k T_{1}}{2 !}\right) Q *\left[\frac{\nabla T}{k}\right)^{2}-\frac{\sigma^{2}}{2 ! D_{1}}\right] X_{o}^{\prime \prime}+\left[\frac{(\nabla T)^{2}}{0 !}+\frac{(\nabla T)^{3}}{1 ! k}\right] X_{o}^{\prime}=0
\end{aligned}
$$

It becomes apparent that if $\sigma^{2}, X_{\mathrm{o}}$ and $X_{\mathrm{o}}^{\prime}$ are known, then every higher derivative of $X_{\mathrm{o}}$ may be found uniquely from the sequence of equations. If only the steady state solution is needed, then the values of $X_{\mathrm{o}}$ and $X_{\mathrm{o}}^{\prime}$ are easily found from the boundary conditions and $\sigma=0$, but finding the transient solution which includes a non-zero constant, $\sigma^{2}$, is a more challenging task. 
An approach for determining $\sigma^{2}$ is to realize that this parameter represents an eigenvalue and that there is an infinite set of constants that will satisfy the conditions of the problem. One method for obtaining them is to expand $X$ in an orthogonal series such as a Fourier or Tschebyshev series, differentiate and substitute into Eq.(B-5), (B-9), use the boundary conditions of the problem to define the eignefunction that must be satisfied by the eigenvalues, and use the orthogonality of the expansion functions and the initial condition to evaluate the coefficients. This is a very laborious process, and it is ill suited to the present application, not only because of its difficulty, but because of the large numbers of terms that must be included in the series similar to that of Eq.(B-6) for convergence under many circumstances. Hence, I opt for the analytical solution presented in the main body.

For the time dependence, I note that in the classical diffusion transient, the time constant, $\tau_{D}$, is independent of the magnitude of the driving force, $\nabla n$. The influence of the Soret effect is to simply alter the magnitude of the driving force, hence it should not influence the characteristic time for diffusion. The nonuniformity of the diffusivity because of the thermal gradient causes a spatial variation in the local characteristic diffusion time, but by using the average value of diffusivity as described in the main body, the result for the overall thickness will be very nearly correct. 


\section{APPENDIX C - ITER CDA DIVERTOR LOADING ESTIMATES}

One really needs to use multi-dimensional calculation results such as those given by the Braams code ${ }^{\mathrm{C} 1}$ in developing an expression for the particle and energy flux distributions over the divertor. Such codes contain many sophisticated and interactive parameters. In the absence of detailed results for a particular configuration, it is advantageous to have an approximation that will provide general agreement with the complex codes. In this appendix I show how that was done for the ITER CDA divertor.

Results from 2-D calculations have the appearance of an exponential decay in both heat flux and ion flux densities with distance from the strike point for planar divertors. I will resort to published 2-D values of peak and total heat fluxes, peak ion fluxes, and distribution widths to generate a consistent set of total particle and heat load distributions for the outboard divertor plate segment. ${ }^{\mathrm{C2}}$ Inboard loadings will be extrapolated from these. Estimates of key parameters are provided in Table C-1. ${ }^{\mathrm{C3}}$ The basic structure of the metallic face is assumed to be something like that shown in Figure C-1. The divertor poloidal geometry profile assumed is shown in Figure C-2. Note that not all material combinations listed in the figure are compatible. Niobium would not be used as a backing material for beryllium, for example. In what follows I have assumed that the plasma-facing material is beryllium with a copper supporting structure. Permeation and retention in beryllium so dominate that for the overall system that the beryllium may be considered independently from the copper.

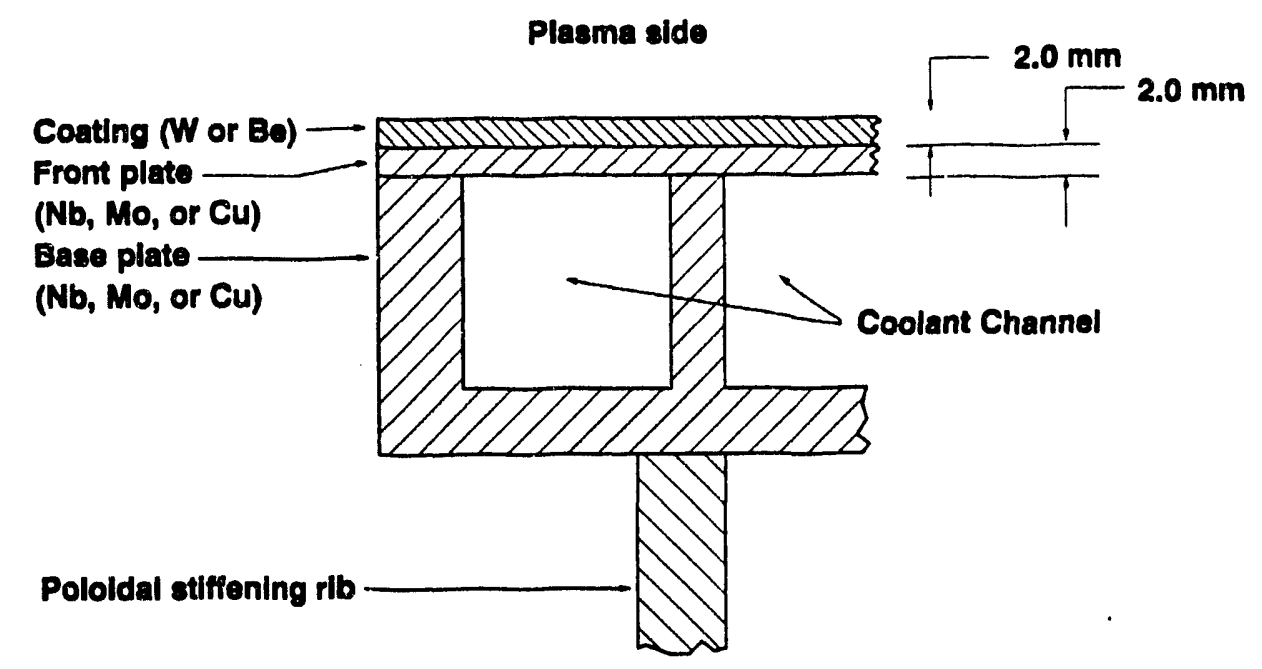

Support structure

Figure C-1. Typical ITER CDA divertor plate cross section. 

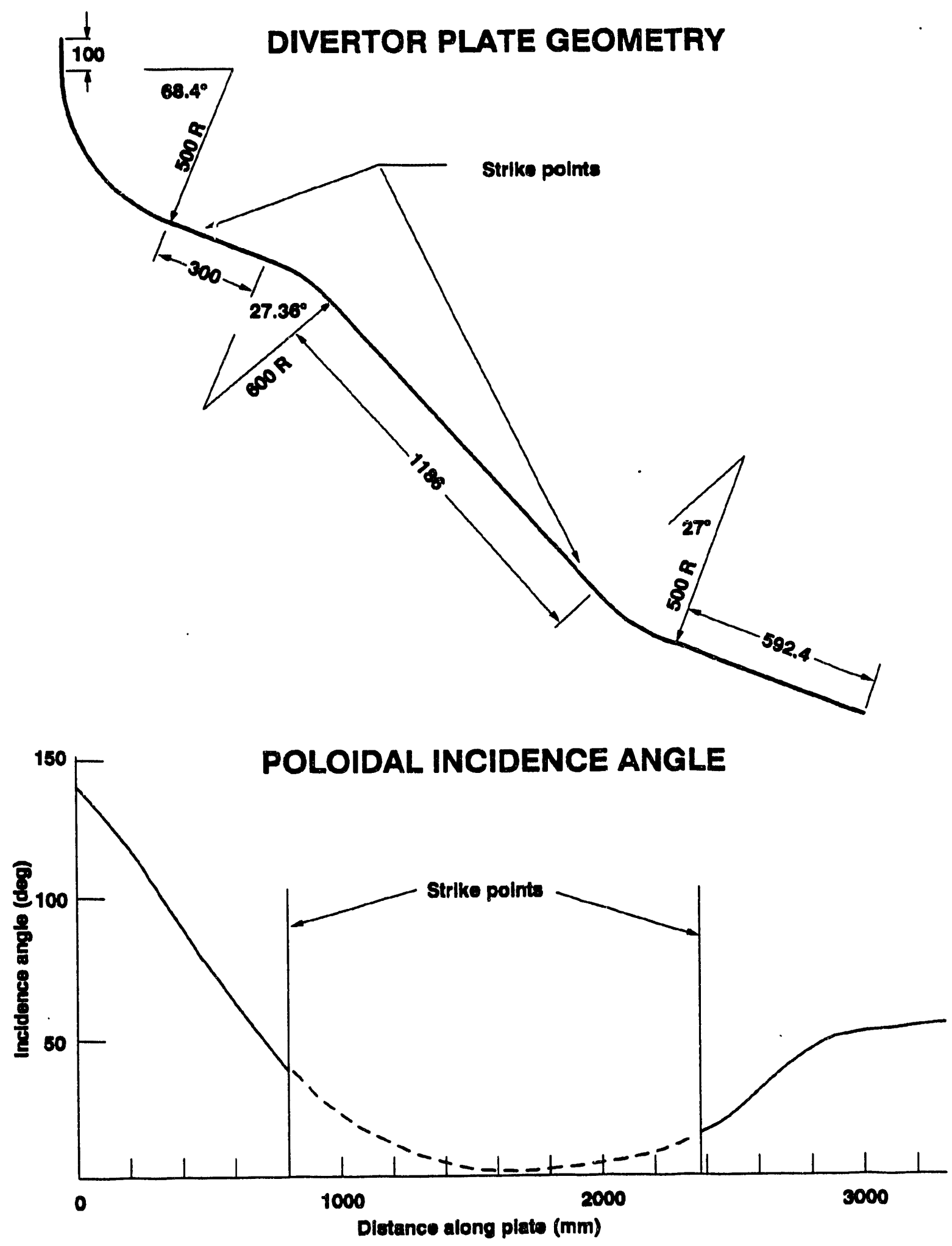

Figure C-2. Geometric description of divertor plate used in calculating ion and heat flux loads. 
Table C-1. Physics data pertinent to loading calculations for the ITER CDA divertor. ${ }^{\mathrm{C2}}$

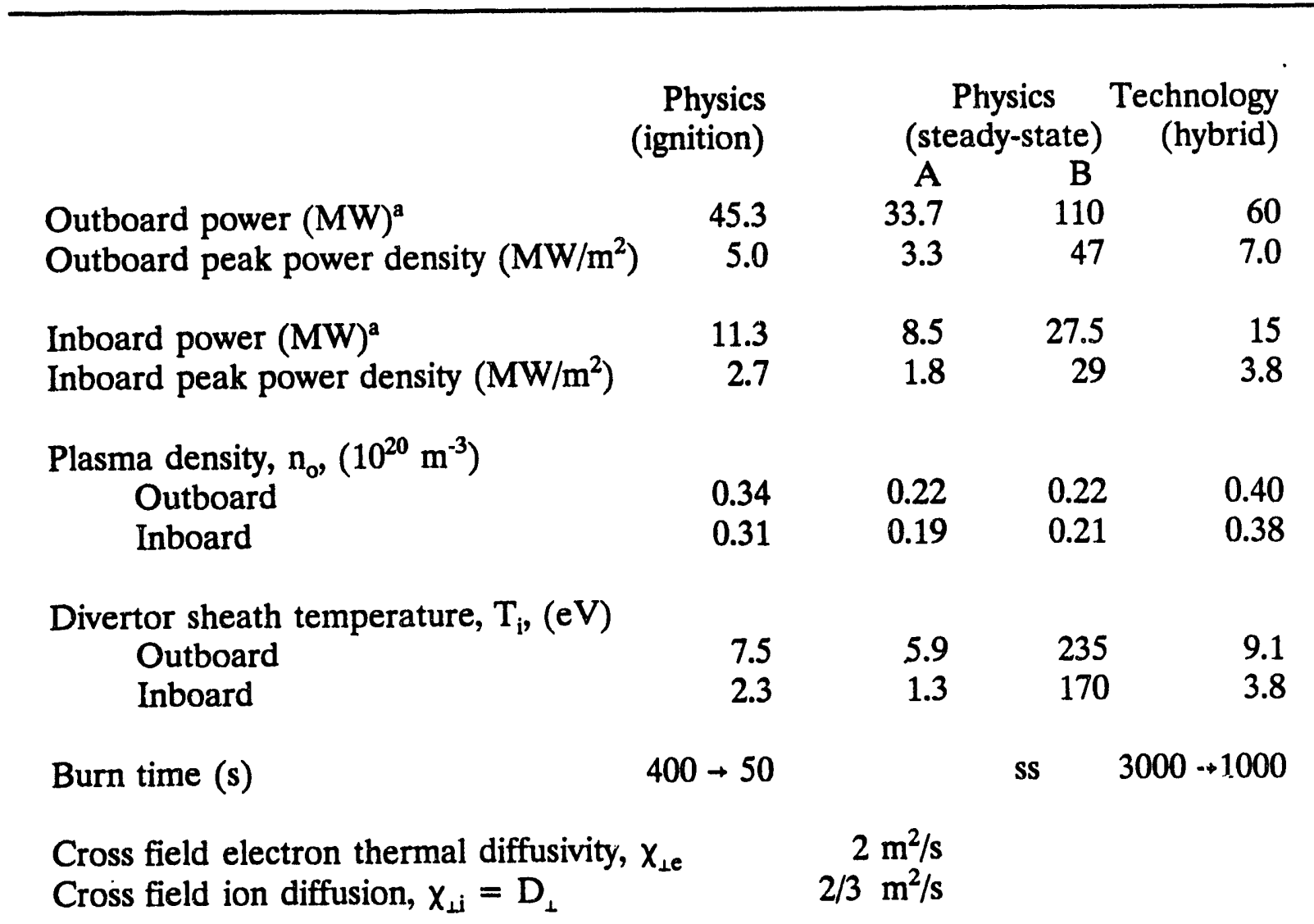

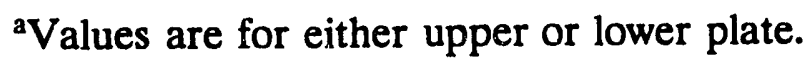

\section{C.1 Flux Distributions}

For the spatial distribution of fluxes over the surface, I first map distance $\mathbf{x}$ away from the separatrix, perpendicular to the magnetic flux surfaces, and normalized by the magnetic field intensity onto the dimension, $s$, in the poloidal plane along the surface of the divertor. $I$ assume $B$ is effectively uniform at the divertor plate. Hence for distance, $s$, measured away from the strike points along the divertor plate's intersection with the poloidal plane,

$$
x(s)=\frac{B_{d}}{B_{m}} \oint_{0}^{s} \sin \theta_{p} d l
$$

where $B_{\mathrm{d}}$ is the intensity of the magnetic field at the divertor, $B_{\mathrm{m}}$ is the corresponding flux intensity at the midplane, and $\theta_{p}$ is the poloidal angle the magnetic field makes with the surface (see Figure C-2). I take $\frac{B_{d}}{B_{m}}$ to be 0.21 for the outboard plate and 0.2 for the inboard. The particle flux density at the divertor surface is then 


$$
J_{i}(s)=J_{o} \exp \left(-\lambda_{n} x(s)\right) \frac{B_{d}}{B_{m}} \sin \theta_{p}(s) .
$$

Here, $J_{\mathrm{o}}$ is the peak value of particle flux density, referred to the midplane, that will be determined from the 2-D code results. From transport considerations, ${ }^{\mathrm{C} 4} \lambda_{\mathrm{n}}$ should vary as $\mathrm{T}^{1 / 4}$, and because

$$
J_{1 i}=\frac{1}{2} n_{i} v_{i}
$$

where $v_{\mathrm{i}}$ is the ion thermal velocity, the flux should scale linearly with plasma density, $\mathrm{n}$, and with the square root of temperature, $T$, at the divertor sheath (see Eq.(C-15) below).

The value of $\lambda_{n}$, referred to the midplane, is obtained from Ref.C5 as $76.3 \mathrm{~m}^{-1}$ for $\mathrm{n}=6.7 \times 10^{20} \mathrm{~m}^{-3}$ and $\mathrm{T}=12 \mathrm{eV}$ by assuming an e-folding length of $27 \mathrm{~cm}$ on a flat plate inclined at $15^{\circ}$ from the separatrix and a magnetic field strength ratio of 0.214 . Hence, I assume that

$$
\lambda_{n}=76.3\left[\frac{T(\mathrm{eV})}{12}\right]^{\frac{1}{4}}\left(\mathrm{~m}^{-1}\right) \text {. }
$$

Then, from ${ }^{4}$

$$
\lambda_{T}=\lambda_{n}\left[\left[\sqrt{\frac{1}{4}\left(\frac{3 D}{\chi}+1\right)^{2}+(2 \gamma-3) \frac{D}{\chi}}-\frac{1}{2}\left(\frac{3 D}{\chi}+1\right)\right]\right.
$$

and

$$
\gamma_{e}=1+\frac{1}{4} \ln \left(\frac{m_{i} T_{e}}{m_{e} T_{i}}\right)
$$

with $T_{\mathrm{i}}=T_{\mathrm{e}}$ and an ion mass of $2.5 \mathrm{amu}$,

$$
\lambda_{t}=0.655 \lambda_{n} \text {. }
$$

Again from Ref.C5, I take the peak particle flux, $J_{\mathrm{o}}$, as $5.0 \times 10^{23} \mathrm{~m}^{-2} \mathrm{~s}^{-1}$. Referred to the midplane that becomes $9.2 \times 10^{24} \mathrm{~m}^{-2} \mathrm{~s}^{-1}$, so that for other conditions,

$$
J_{0}=9.2 \times 10^{24}\left[\frac{n\left(10^{20} \mathrm{~m}^{-3}\right)}{6.7}\right] \sqrt{\frac{T(\mathrm{eV})}{12}}\left(\mathrm{~m}^{-2} \mathrm{~s}^{-1}\right) \text {. }
$$


The correspondirig heat intensity function, $q(x)$, based on

$$
\frac{d q_{\perp e}}{d x}+\frac{2 \gamma_{e} n_{i} T_{e}}{\tau_{1}}+q_{e i}=0
$$

and

$$
\frac{d q_{\nu i}}{d x}+\frac{2 n_{i} T_{i}}{\tau_{i}}-q_{e i}=0
$$

noting that $\gamma=\gamma_{e}+1$, is

$$
q(x)=2 \gamma T(x) \nu_{i}(x)
$$

where $\mathrm{T}(\mathbf{x})$ comes from

$$
T=T_{o} \exp \left(-\lambda_{T} x\right)
$$

That suggests the scaling law for 2-D hedt fluxes shou' $\mathrm{d}$ be

$$
q(x)=q_{0} \exp \left[-\left(\lambda_{n}+\lambda_{t}\right) x\right]
$$

where $q_{0}$ is the peak value found in the 2-D results. Brsth total power to inner and outer SOLs and peak heat fluxes to each divertor plate segmert are specificd in Ref.C2 as are plasma densities and temperatures at the inner and outer aivertor sheaths (see Table C-1). This scaling using peak hent fluxes as given in Table $\mathrm{C}-1$ results in total heat ioads to the inboard and outboard divertor plates that are in reasonable agreement with the values listed in Table C-1.

An example of the resulting distributions is given in Figure C-3(a) anu (b). Figure C.3(a) is the heat flux profile given as a function of distance from the inboard end of the divertor plate while Figure C-3(b) shows the corresponding ion flux density distribution for the Technology Phase parameters.

\section{C.2 Implantation Depth}

Having arrived at a reasonable estimate for the particle and heat flux distributions, the next step is to determine the implantation depth profile. I assume that the particles entering the divertor plate do so in the average direction of velocity vector incidence and that they are accelerated into the surface by a sheath potential which is taken as $2.5 \mathrm{kT} / \mathrm{e}$, where $T$ is the local plasma temperature.

The incidence angle of the magnetic field lines will vary with location on the divertor plate according to 


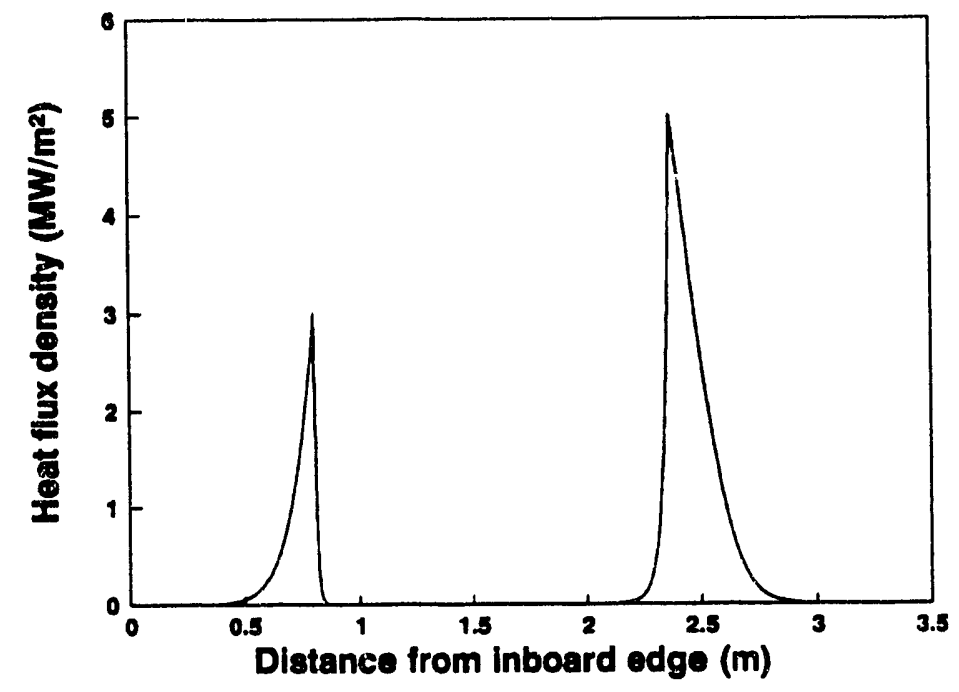

(a)

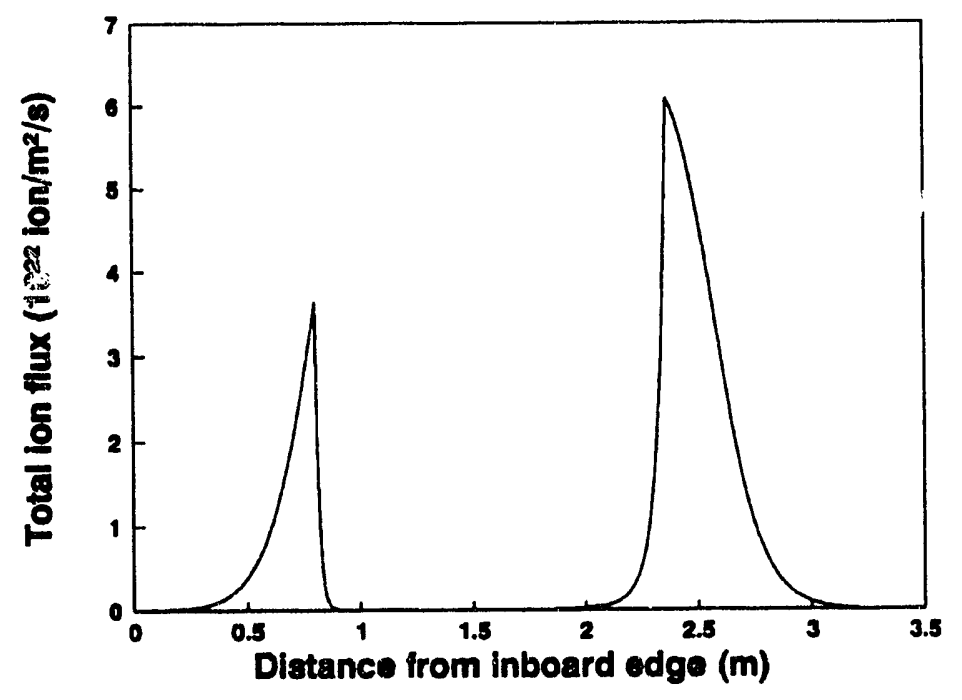

(b)

Figure C-3. Heat and particle flux distributions calculated for the ITER CDA divertor in the Technology Phase. 


$$
\theta=\cos ^{-1}\left[\frac{\cos \theta_{p}}{\left[\sqrt{\left.\frac{R q}{a}\right)^{2}+1}\right.}\right]
$$

where $R$ and $a$ are the major and minor radii for the plasma and $q$ is the safety factor, but for simplicity, in this part of the analysis the inclination angles, $\theta_{\mathrm{p}}$, are assumed constant at $40^{\circ}$ for the inboard plate and $15^{\circ}$ for the outboard plate.

To get the average incidence angle of the ion velocities with respect to the unit normal to the surface, I assume that ion velocity components are given by

$$
\begin{aligned}
& v_{1}=\sqrt{\frac{k T}{m}} \\
& v_{\perp}=\sqrt{\frac{2 k T}{m}}
\end{aligned}
$$

and that the incidence angle, $\alpha$, for the ions is

$$
\alpha=\cos ^{-1}\left(\frac{v \cdot n}{v}\right)=\cos ^{-1}\left[\frac{\cos (\theta)}{\sqrt{3}}+\left[\overline{\frac{2}{3}} \sin (\psi) \sin (\theta)\right]\right.
$$

where $\theta$ is the angle made by the magnetic field line with the unit normal, $\mathbf{n}$, and $\psi$ is the angle of $v_{1}$ with respect to the reference orientation, in the direction of $B \times n$. The average value of $\alpha$ is then given by

$$
<\alpha(\theta)>=\frac{1}{\pi} \int_{0}^{\pi} \alpha(\theta, \psi) d \psi
$$

which I solved using 10-point Gaussian quadrature. For the assumed geometry, $\theta$ has values of $85.5^{\circ}$ and $88^{\circ}$ for the inboard and outboard divertor plates, respectively. The corresponding values for $\langle\alpha\rangle$ are $53.8^{\circ}$ and $56.7^{\circ}$.

I used the TRIM code ${ }^{6}$ to generate implantation depth values for the various segments of the divertor plate. I assume the ions striking the divertor plate have three times their thermal energy ${ }^{7}$ and that they are monoenergetic. I assume a lattice displacement energy of $25 \mathrm{eV}$ and a surface binding energy of $2 \mathrm{eV}$ in the TRIM calculations. Temperature distributions are taken to be 


$$
T(x)=T_{o} \exp \left(-\lambda_{T} x\right)
$$

with $\lambda_{\mathrm{T}}$ as given by Eq.(C-5) The implantation depth in Angstroms for tritium into beryllium is found by that process to be 15 times the plasma temperature, $\mathrm{T}$ in $\mathrm{eV}$. The TRIM code indicated backscattering could be as high as $46 \%$, but this was ignored in the calculations done. It could be included by reducing the particle load values.

The divertor is logically divided into inboard and outboard regions with each region further divided into two zones by the separatrices. Each of these four zones is further subdivided into up to five segments by $\left(\lambda_{n} \mathrm{x}\right)$ reaching values of $0.223,0.511,0.916$, and 1.609. Only four segments were used in regions outboard of the strike points. Table $\mathrm{C}-2$ lists the various segment parameters for the Technology Phase. These values were used as inputs for the calculations reported in the main part of the report.

Table C-2. Parameters for ITER CDA Technology Phase used in calculations.

\begin{tabular}{|c|c|c|c|c|c|}
\hline Segment & $\begin{array}{c}\text { Location } \\
(\mathrm{m})\end{array}$ & $\begin{array}{l}\text { Aręa } \\
\left(\mathrm{m}^{2}\right) \\
\end{array}$ & $\begin{array}{c}\text { Particle Load } \\
\left(\mathrm{DT} / \mathrm{m}^{2} \mathrm{~s}\right)\end{array}$ & $\begin{array}{l}\text { Heat Flux } \\
\left(\mathrm{MW} / \mathrm{m}^{2}\right)\end{array}$ & $\begin{array}{l}\text { Plasma } \\
\text { Temp (eV) }\end{array}$ \\
\hline $\begin{array}{r}1 \\
2 \\
3 \\
4 \\
5 \\
6 \\
7 \\
8 \\
9 \\
10 \\
11 \\
12 \\
13 \\
14 \\
15 \\
16 \\
17 \\
18\end{array}$ & $\begin{array}{r}0-.0 .61 \\
0.61-0.68 \\
0.68-0.73 \\
0.73-0.77 \\
0.77-0.80 \\
0.80-0.81 \\
0.81-0.82 \\
0.82-1.60 \\
1.60-2.26 \\
2.26-2.32 \\
2.32-2.34 \\
2.34-2.36 \\
2.36-2.37 \\
2.37-2.43 \\
2.43-2.49 \\
2.49-2.56 \\
2.56-2.66 \\
2.66-3.30\end{array}$ & $\begin{array}{r}14.82 \\
1.76 \\
1.28 \\
1.03 \\
0.78 \\
0.26 \\
0.26 \\
21.73 \\
20.34 \\
1.64 \\
0.66 \\
0.66 \\
0.33 \\
1.98 \\
2.01 \\
2.36 \\
3.41 \\
23.00\end{array}$ & $\begin{array}{l}1.89 E+21 \\
1.53 E+22 \\
2.43 E+22 \\
3.30 E+22 \\
3.97 E+22 \\
1.30 E+20 \\
4.60 E+17 \\
2.16 E+13 \\
4.77 E+16 \\
3.84 E+19 \\
7.42 E+20 \\
1.66 E+21 \\
1.03 E+22 \\
2.41 E+22 \\
2.39 E+22 \\
2.12 E+22 \\
1.55 E+22 \\
2.26 E+21\end{array}$ & $\begin{array}{r}0.022 \\
0.397 \\
0.883 \\
1.513 \\
2.209 \\
1.7 \mathrm{E}-04 \\
1.5 \mathrm{E}-08 \\
1.9 \mathrm{E}-14 \\
1.4 \mathrm{E}-08 \\
1.7 \mathrm{E}-04 \\
0.015 \\
0.079 \\
1.012 \\
4.296 \\
3.607 \\
2.614 \\
1.369 \\
0.091\end{array}$ & $\begin{array}{l}0.73 \\
2.37 \\
2.87 \\
3.27 \\
3.62 \\
3.10 \\
2.57 \\
0.27 \\
0.52 \\
5.64 \\
7.05 \\
7.99 \\
8.54 \\
8.53 \\
7.83 \\
6.96 \\
5.69 \\
1.52\end{array}$ \\
\hline
\end{tabular}

aMeasured from inboard edge. 


\section{APPENDIX C REFERENCES}

C1 G. J. Braams, M. F. A. Harrison, E. S. Hotston, and G. Morgan, in Proc. 10th INt. Conf. on Plasma Physics and Controlled Nuclear Fusion Research, London, September, 1984.

C2 M. F. A. Harrison and E.S. Hotston, "Edge Plasma and Surface Conditions in ITER," Journal of Nuclear Materials 176 \& 177 (1990) 256-261.

C3 Revised Section 3, Physics; Design Information Document, ITER-IL-SA-6-0-4, May $17,1990$.

C4 H. C. Howe, "Physics Considerations for the FED Limiter," ORNL/TM-7803, July 1981.

C5 S. A. Cohen et al., "Two-Dimensional Fluid Simulations of the ITER SOL Plasma," Journal of Nuclear Material 176 \& 177 (1990) 909-915.

C6 J. F. Ziegler, "TRIM-90, The Transport of Ions in Matter (Version 90.xx), IBMResearch, Yorktown, NY, USA, June 11, 1990.

C7 P. C. Stangeby and G. M. McCracken, "Plasma Boundary Phenomena in Tokamaks," Nuclear Fusion 30, No. 7 (July 1990) pp. 1225-1287. 

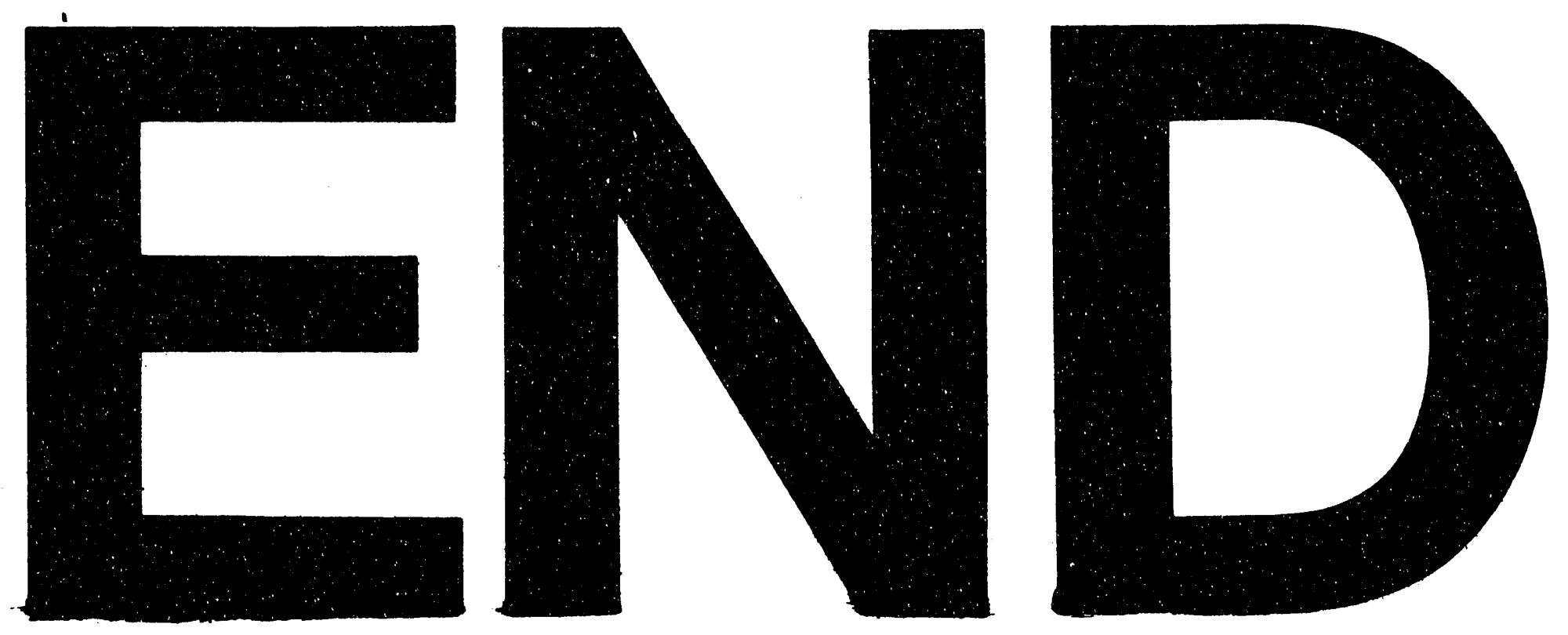

.
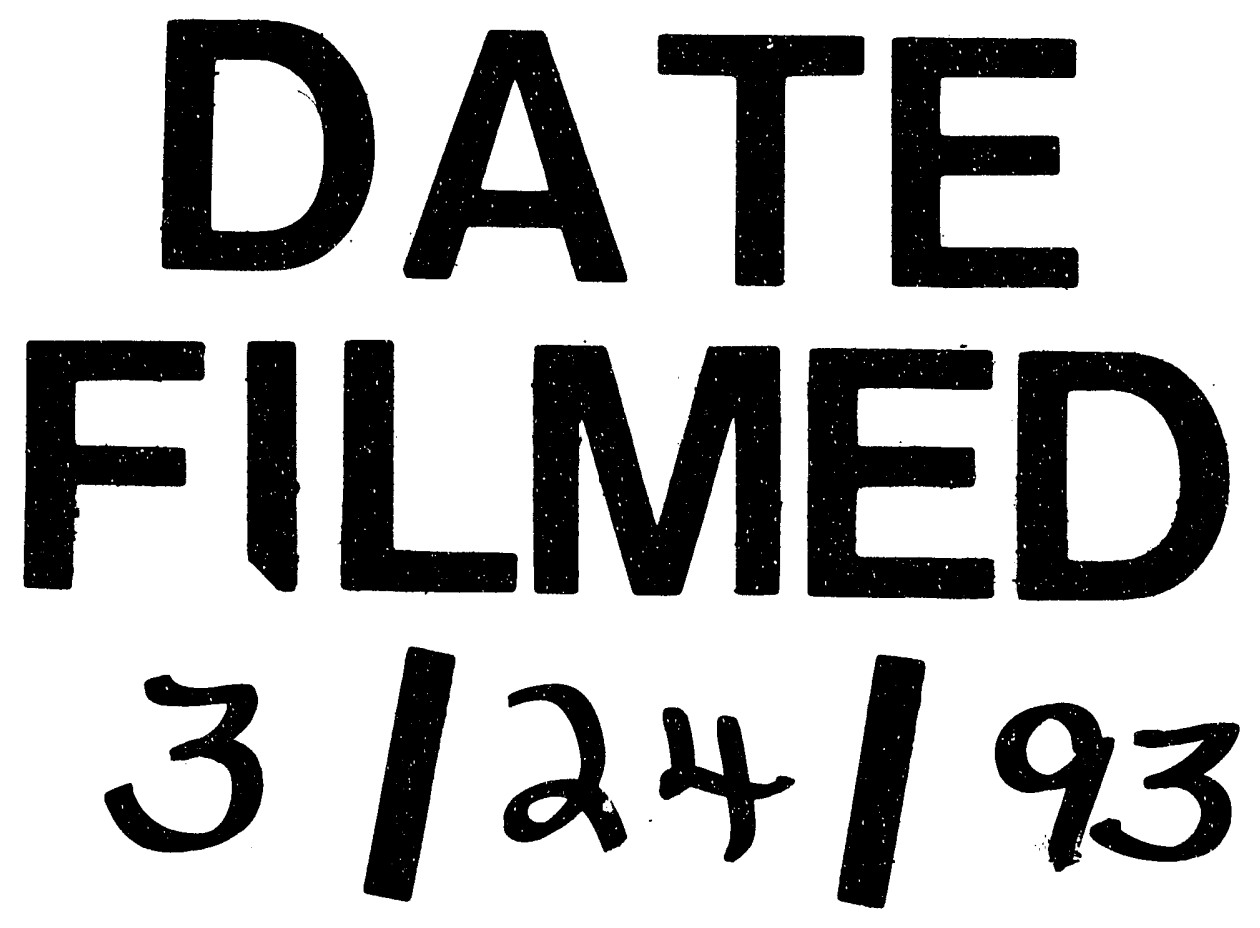
